The final version of this paper appeared in Pattern Recognition Journal, vol. 28, no. 2, pp. 171-198, 1995.

\title{
The Construction of Labeled Line Drawings from Intensity Images $^{1}$
}

\author{
Deborah Anne Trytten \\ School of Computer Science \\ University of Oklahoma \\ Norman, OK 73019-0631, USA \\ dtrytten@mailhost.ecn.uoknor.edu \\ Fax: $405-325-4044$ \\ Mihran Tuceryan \\ European Computer-Industry Research Centre (ECRC) \\ Arabellastraße 17 \\ 81925 München, Germany \\ mihran@ecrc.de \\ Fax: +(49) (89) 92699-170
}

June 27, 1994

\footnotetext{
${ }^{1}$ This research was supported in part by the National Science Foundation grants IRI-9103143 and CDA8806599. The facilities of the Pattern Recognition and Image Processing Laboratory at the Michigan State University are also gratefully acknowledged.
} 


\begin{abstract}
This paper describes the POLL (Perceptual Organization and Line Labeling) system for obtaining labeled line drawings from single intensity images using an integrated blackboard system. This system emphasizes the data driven extraction of $3 D$ geometric information from intensity images. The robustness of the system comes from it being implemented in an integrated framework in which the errors made by one module can be diagnosed and corrected by the constraints imposed by other modules. The system is able to generate an initial line drawing from an intensity image in the domain of piecewise smooth objects.

Four modules were used as knowledge sources: weak membrane edge detection, curvilinear grouping, proximity grouping, and curvilinear line labeling. An initial representation of the image data is built using the first three knowledge sources. This representation is analyzed using a modified curvilinear line labeling algorithm developed in this paper that uses figureground separation to constrain legal line labelings. This modified line labeling algorithm can diagnose problems with the initial representation. The modified line labeling algorithm can find errors such as missing edges, improperly typed vertices, and missing phantom junctions. Errors in the representation can be fixed using a set of heuristics that were created to repair common mistakes. If no irreparable errors are found in the representation, then the modified line labeling algorithm produces a $3 D$ interpretation of the data in the input image without explicitly reconstructing $3 D$ shape.
\end{abstract}




\section{Introduction}

One of the main goals of computer vision is to extract spatial and geometric information about the external world seen in an image. The extracted spatial information could be as simple as identifying empty spaces for navigational or obstacle avoidance purposes, or it could be for manipulation or object recognition purposes.

Object recognition is one of the most important and frequently researched topics in computational vision, due to its potential for wide applicability to important automation problems. There are many proposed methods in the literature for accomplishing object recognition. One very common approach is to match a representation of an object that was derived from image data to $3 D$ descriptions of objects stored in a model database. Such $3 D$ descriptions can also be used for purposes other than recognition, such as manipulation.

In an intensity image, the light received by the camera is a function of several factors such as the shape and reflective properties of the surfaces, the number, type, and relative direction of light sources, and the viewing direction. These factors are highly interrelated making the direct recovery of the $3 D$ shape of the imaged objects difficult. Therefore, the extraction of a $3 D$ description from an image involves an inference process. There is a broad literature on a set of techniques for inferring such information based on explicit reconstruction of the $3 D$ shape. These include shape from shading $[1,2,3,4]$, shape from texture $[5,6,7,8]$, shape from motion [9], shape from stereo $[10,11]$, and many other similar processes collectively referred to as "shape from X." While these algorithms are gaining mathematical rigor [9], they still lack the robustness that is necessary to be useful in a general environment. Other approaches include extraction of qualitative $3 D$ information from images. An early example of such a process is extracting line drawings from images with possible $3 D$ intrepretations attached. This is attractive because it is boundary based and terse. Boundaries have been shown to be useful in human vision [12]. The problem is that it is difficult to reliably extract the perfect line drawings from images which are necessary for most line labeling algorithms to work properly.

Another approach to extracting qualitative information from images involves inferring structure through perceptual organization. This line of research has been motivated by cues from the human visual system [13]. The human visual system can organize and interpret images even when $3 D$ shape cannot be reconstructed. To do this, the salient features of the image, called tokens, need to be identified, organized, and interpreted. The capability of the human visual system to group tokens together in a meaningful way without any prior knowledge of the contents of the image, is called perceptual organization. Perceptual organization collects tokens in the image plane together into significant groupings using only general rules. These tokens can be 
recursively grouped, or subdivided as the scene is further organized. Cooperating and competing groupings are explored until a globally good organization of the image is found. Groupings of tokens can then be used to infer $3 D$ properties [14]. The problem with this approach is that often there are many competing plausible groupings. Deciding among these competing groupings without the proper $3 D$ context is difficult.

This paper will describe the development of the POLL system (Perceptual Organization for

Line Labeling) which uses the processes described above in an integrated manner, to find labeled line drawings from single monochromatic intensity images. Our motivation was:

- to reduce the fragility of each of these individual processes through the use of multiple cooperative processes which complement each other, and

- to minimize the need for human intervention in this processing

The organization of the paper is as follows. Section 2 will give a brief review of the background and previous work. Section 3 will describe the overall architecture of the POLL system. Section 4 will explain how the initial analysis of image information is performed with the perceptual knowledge sources. Section 5 will give the method proposed to discern images that have been properly analyzed by the initial processing from those that have not using the diagnostic and interpretive knowledge source. Once inconsistencies in the initial representation of the image have been found, Section 6 shows how some of these defects can be corrected using the control and repair knowledge sources. Section 7 will present experimental results. The final section, Section 8, will list the contributions of the paper and suggest related topics for further research.

\section{Background}

The POLL system's goal is to extract labeled line drawings from intensity images in an integrated framework. The modules used in the implementation of the POLL system are (a) low level modules, (b) perceptual organization modules, and (c) a $3 D$ interpretation module in the form of line labeling. We first give a brief review of previous work done which is relevant to the components of the POLL system.

The low level processing component entails the processes that operate directly on the image and produce results in the image domain. It includes such modules as edge detection, segmentation, corner detection, etc. There has been a great amount of research done in the individual modules of this level and there is a vast literature about it. The interested reader can refer to any standard vision textbook $[15,16]$. 
Another module which is integral to the operation of POLL is the perceptual grouping module. Perceptual grouping is the organization by the human visual system of image tokens in a scene into meaningful groupings without using any domain specific knowledge or without any explicit shape information being present. An appreciation for this aspect of the human visual system dates back to the Gestalt school of psychology [17], which identified the first principles of what is now known as perceptual organization. These principles include proximity, similarity, good continuity, closure, symmetry, and figure-ground separation.

In computational vision, the importance of perceptual organization was brought to the forefront by Witkin and Tenenbaum [18] and Lowe [14]. Witkin and Tenenbaum analyzed the importance of structure, meaning spatiotemporal coherence or regularity, in visual perception. They argued that when regular structure is observed, it is significant because it would have been unlikely to have arisen accidentally. Lowe sharpened this idea in [14] when he proposed that the significance of a grouping of tokens is proportional to how unlikely the relationship between the tokens was to have arisen by accident, called non-accidentalness. Other studies that attempt to model perceptual organization principles computationally include Tuceryan [19], Rosin and West [20], Trytten [21], McCafferty [22], Sarkar and Boyer [23], and Mohan and Nevatia [24].

Labeling of line drawings was originally done in the trihedral world [25, 26], and later extended to general polyhedra [27]. Shadow edges and surface markings were added in [28], and origami objects were used in [29]. A junction catalog for piecewise smooth objects was derived by Malik in [30]. Piecewise smooth objects differ from polyhedra in that the surfaces can be curved and are not restricted to be planar. There have been various attempts in the past at extracting labeled line drawings from real images with varying levels of generality and varying degrees of success $[31,32,33,34,35]$.

Another major aspect of the POLL system is the integration of various visual processing modules. Integration has become an important research topic in computational vision [36, 37 , $38,39,40,35]$. The primary goal of integration is improving the robustness and flexibility of computational vision algorithms.

Moravec's certainty grid [39], Gibbs distribution and Markov Random Field frameworks (MRFs) $[41,38,36,22]$ are examples of integration within a uniform mathematical framework. In these approaches often some sort of optimization process is involved in the form of maximizing a posteriori probabilities or minimizing some energy function. When energy minimization problems are being solved, it is often desirable that the solution found be smooth. Regularization theory is the rigorous approach to smoothness as a constraint which have been suggested and used by various researchers $[42,43,44]$. 
Any integration strategy that does not exhibit the uniformity of the integration strategies mentioned above will be classified as non-uniform. This approach is often used for integrating pairs of processes. Many of these schemes take advantage of the strong constraints in the specific domain under consideration. Examples of this approach include works by Ahuja, et al. [45, 6] and Malik and Maydan [46].

When the number of processes to be integrated becomes large, creating a non-uniform system is more difficult. If this integration is handled informally, chaos can result. A useful structure for integrating diverse processes which work on a common problem is the blackboard system $[47,48]$. Blackboard systems have previously been used for image processing and computer vision $[37,49]$. Work is also being done on specialized architectures for blackboards, concurrency and parallelism, and real time blackboard systems [50].

\section{The POLL System}

This paper will describe the construction of the POLL system (Perceptual Organization and Line Labeling) that can find labeled line drawings from single intensity images. The input into the system will be a single intensity image of one or more objects in a domain which will be precisely described shortly. The output from the system will be a line drawing labeled with a $3 D$ interpretation, and a database of intermediate representations and partial results that may be useful for further processing. Finding labeled line drawings is a very difficult problem, and there will be some images which are not correctly processed. In these images, it is desirable to obtain intermediate information that could be used by other processing modules to work towards the final goal of $3 D$ interpretation.

Visual perceptual organization and $3 D$ interpretation do not consist of a single cohesive process, but rather of a diverse collection of processes working towards the goal of interpreting images. For these perceptual processes to be able to cooperate and compete to find meaningful relationships within the image, the processes need to be integrated. Integrated processes can use each others constraints and expertise to guide the search for good groupings.

An example of the need for integration is shown in Figure 1. This very simple polyhedral scene would create enormous difficulties for existing line labeling methods. The imaged object is within both the domain of polyhedral objects and the domain of piecewise smooth objects. But it is unlikely that any edge detection scheme could recover the front corner edge of this object, since edge detection relies upon spatial variation in intensities and there is no spatial variation in intensity values across the front edge of the prism. Line labeling modules require perfect input and cannot recover from edge detection mistakes. And edge detection modules cannot 


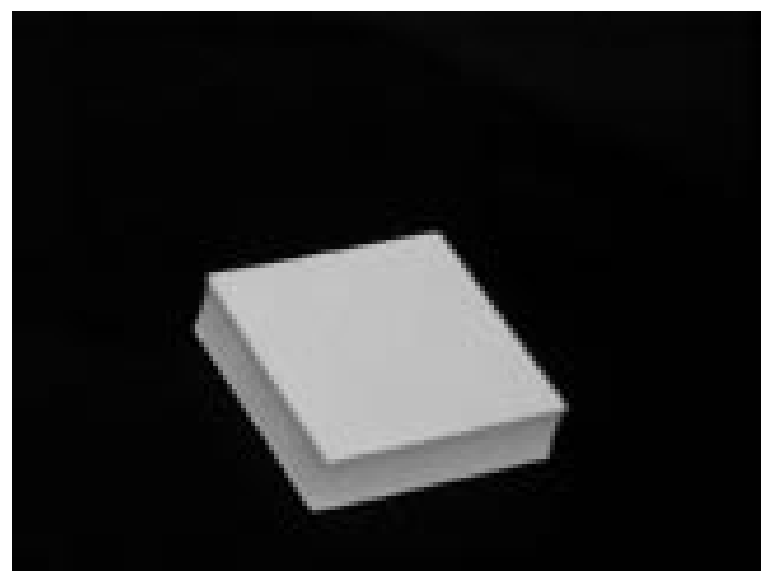

Figure 1: An intensity image of a simple square block. Notice that there is almost no variation in intensity across the front corner of the block. This makes it difficult for edge detection to find this edge without finding other noise edges.

give perfect edges. Therefore, even an unavoidable edge detection failure as in this example will result in a failure of line labeling.

The selection of the integration framework will depend upon the nature of the modules and upon the nature of the information each module processes. If all the modules are working on information in the same representation scheme, then using uniform integration frameworks becomes easier but not obligatory. This still does not mean that uniform schemes are the most appropriate to use in these cases. On the other hand, if the way in which the information is represented lacks a uniformity to begin with, then it becomes harder to justify and also implement such uniform integration schemes.

The integration framework used is the blackboard system [47]. A blackboard system uses a common database, called the blackboard, to hold input, partial solutions, and control data. These data objects are processed by independent knowledge sources, each of which specializes in one type of processing. These knowledge sources evaluate the current blackboard configuration, look for opportunities to contribute to improving the solutions, and perform their specialized processing. The results of this processing are left on the blackboard for other knowledge sources to opportunistically use.

Blackboard systems have significant advantages over other integration frameworks for this research. Blackboard system modules can be altered without undue hardship since knowledge source independence is maintained. This is important since there may well be significant improvements in some of the modules in the future. This type of flexibility permits a system to evolve as the understanding of computational vision increases. The blackboard has an independent control module that can strategically explore the space of solutions. Bringing these control issues to the forefront, offers an opportunity to examine the interaction among the various mod- 
ules. Methods without elaborate control structures cannot provide this type of introspection into their processing.

Figure 2 shows the overall structure of the POLL system. The knowledge sources are divided into four groups. The first group are the low level (perceptual) knowledge sources. These knowledge sources bootstrap the initial line drawing using low level processing and perceptual organization. They are also available to do specialized work for other knowledge sources, and make other contributions to the blackboard database. The diagnostic and interpretive knowledge source is a modified line labeling paradigm that can examine a line drawing and locate inconsistencies. If there are no inconsistencies, a $3 D$ interpretation of the line drawing will be found. The information produced by the diagnostic and interpretive knowledge source is available for the control knowledge source. This knowledge source selects which of the available repair strategies, if any, is suitable for fixing problems with the line labeling. The repair knowledge sources are called by the control knowledge source to fix the line labeling, and in turn call the perceptual knowledge source to reevaluate previous results in light of the the more global diagnostic information that is available. The integration in this system comes from the fact that there is interaction, cooperation, and feedback among the various knowledge sources through communications via the blackboard. Thus, the diagnostic line labeling knowledge source can have a top-down effect on the lower level modules by enforcing constraints in its domain. Notice also that through this type of interaction, there is no fixed, sequential order of processing which must be applied to every image. Depending upon the content and complexity of the image, there may be many different orders of processing and many iterations through the effective feedback loops. This is where the power of integration schemes originates.

The perceptual knowledge sources used in this system are weak membrane edge detection [51], an algorithm that performs edge grouping on the basis of continuity [21], a module that analyzes vertex proximity, and a module that can determine the type of line drawing junctions. A modified line labeling algorithm will be used as the diagnostic and interpretive knowledge source.

The domain of objects that is used in this paper is the set of piecewise smooth objects defined precisely by Malik in [30]. We restrict this domain, however, in order to help control the complexity of the object domain: the number of surfaces that can meet at a vertex in an object in our domain is limited to three. Some examples of real objects that are in the domain and are correctly processed by the POLL system are shown in Figure 3. Examples of objects that are not in this domain are wireframe objects, origami objects, objects where more than three surfaces meet at a junction, a single nappe of a right circular cone. 
Perceptual Knowledge Sources

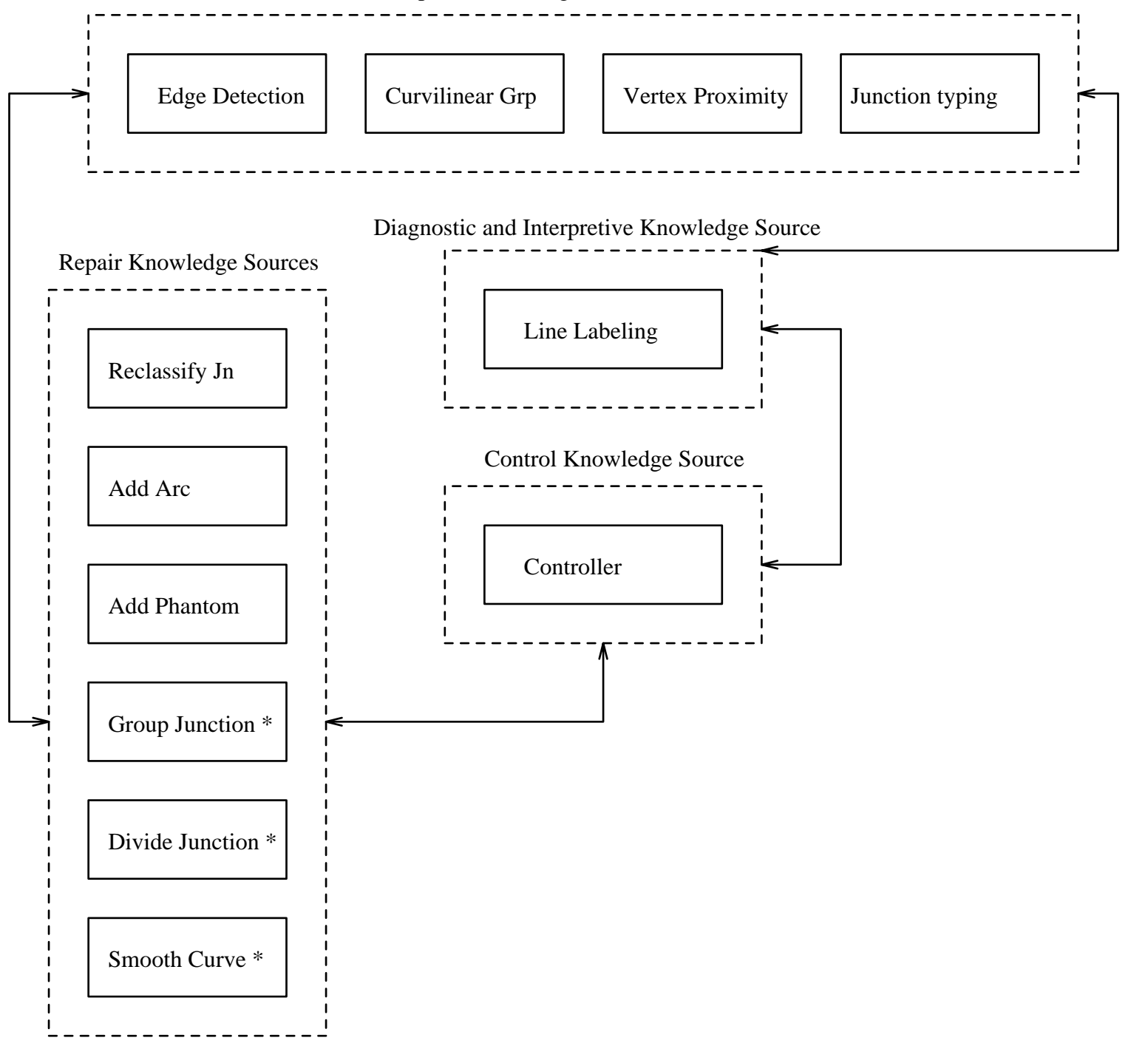

Figure 2: An overview of the POLL system described in this paper. Repair knowledge sources marked with an asterisk have not been implemented.

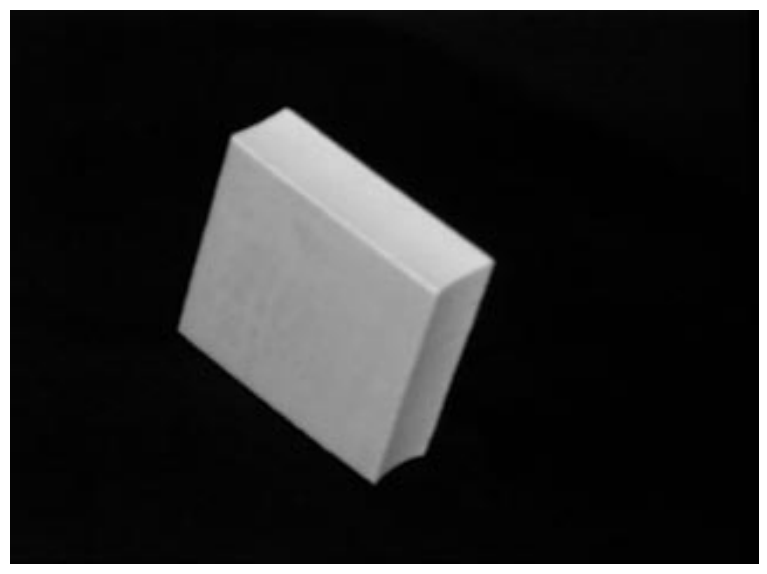

(a)

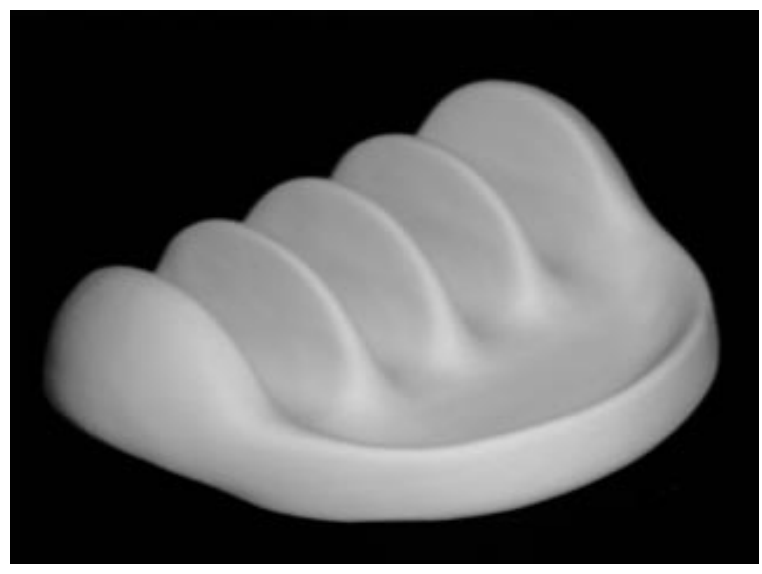

(b)

Figure 3: Two piecewise smooth objects. 
A number of other assumptions must be made for the line labeling algorithm to be applicable. The projection model for the image must be orthographic; the objects must be seen in a general viewpoint; and shadows, texture and markings are not allowed on the objects. These restrictions could be relaxed if a more comprehensive junction catalog were to become available. Below we give a brief description of some of the building block modules of our system.

\subsection{Edge Detection}

The method of edge detection that was selected for this work is fitting a weak membrane model using the Generalized Non-Convexity (GNC) algorithm of Blake and Zisserman [51]. Other edge detection methods we considered include Canny edge detector [52] and other traditional edge detectors. The weak membrane method has a number of advantages over Canny edge detection. We have found that it behaves better at the corners than Canny. This is critical because the corners (junctions) are very important in line labeling.

The weak membrane model is an energy minimization scheme in which a surface is fit to the image data which models a membrane which can have tears (thus modeling discontinuities). The minimization can be done with the usual techniques, but in [51] a deterministic algorithm (GNC) is used which finds the global minimum. For the sake of simplicity, the explanation given below refers to the weak string (the one-dimensional analogue of the weak membrane). The discrete formulation of the energy functional in the weak string is made up of three terms. Let $u_{i}$ be the weak string solution to the functional. Let $x_{i}$ be the initial data. Let $l_{i}$ be a binary function such that $l_{i}=1$ corresponds to a break in the string. The energy functional $E$ to be minimized for the weak string is:

$$
E=\sum_{i}\left(\left(u_{i}-x_{i}\right)^{2}+\lambda^{2}\left(1-l_{i}\right)\left(u_{i}-u_{i+1}\right)^{2}+\alpha l_{i}\right)
$$

The first term penalizes the solution $u_{i}$ for being far away from the data $x_{i}$. The second term penalizes $u_{i}$ for being far from its neighbor. This is a finite element approximation of the first derivative and contributes to the energy only when the string is unbroken. The final term is a penalty for breaks in the string. These three energy penalties together encourage the weak string to be simultaneously close to the data, smooth, and unbroken. The parameters are set to balance smoothness with breaks. If $\lambda$ is large, the string will be heavily penalized for not being smooth, and will tend to break often. If $\alpha$ is large then the string will be broken less frequently, but will smooth over the discontinuities. The two dimensional counterpart of a break in the string is a tear in the weak membrane. The set of tears in the weak membrane correspond to the edges of the detected regions. 
The energy functional needs to be minimized both with respect to $u_{i}$, and with respect to $l_{i}$. This problem is not convex and has the potential to get trapped in local minima during the minimization process. The GNC algorithm is an attempt to avoid this problem in the weak membrane model. This procedure has been shown to converge to the global minimum energy for the weak string, the weak membrane, the weak rod, and the weak plate models if the discontinuities are much further separated than the scale parameter $\lambda$.

One advantage of selecting GNC for the edge detection module is that it tends to find complete boundaries around regions even though this is not guaranteed. It does a good job at the corners and junctions. The weak membrane formulation has only two parameters, both of which have a physical interpretation. These parameters can be changed into another pair of parameters which corresponds to scale and contrast sensitivity which is meaningful for edge detection. The parameters were set initially, after experimentation, and were not altered during the course of processing all the images shown in this paper Appendix A contains a summary of the parameters used in the implementation.

\subsection{Curvilinear Grouping}

Edge detection alone cannot always provide perceptually meaningful lines and curves. Missing, misplaced, and extraneous edges are often detected. The purpose of the curvilinear grouping module is to take the edges that are detected and organize them into more perceptually meaningful structures. This can be done by grouping neighboring image tokens, as perceptual organization would suggest.

Much of the previous research in this area has been dedicated to detecting straight lines $[53,32,54]$ or curves whose model is known a priori $[55,56]$. More general algorithms have also been studied by Lowe, whose algorithm emphasized recursively grouping adjacent edge points into lines at multiple scales [14]. But he used a linear or circular curve model for performing the perceptual organization. In this paper, a more general curvilinear grouping algorithm developed by the authors was used [21]. This algorithm uses good continuity to group neighboring curves. The neighborhood relationship is defined by the perceptually significant Voronoi tesselation [57].

\subsection{Line Labeling}

As previously discussed, there have been many attempts to find a line labeling algorithm in the past. Some of these have widened the domain of objects for which the labeling scheme is defined. Others have attempted to deal with imperfect data. In this paper, we have decided to use a line labeling algorithm with the least restricted object domain currently available. Therefore, 


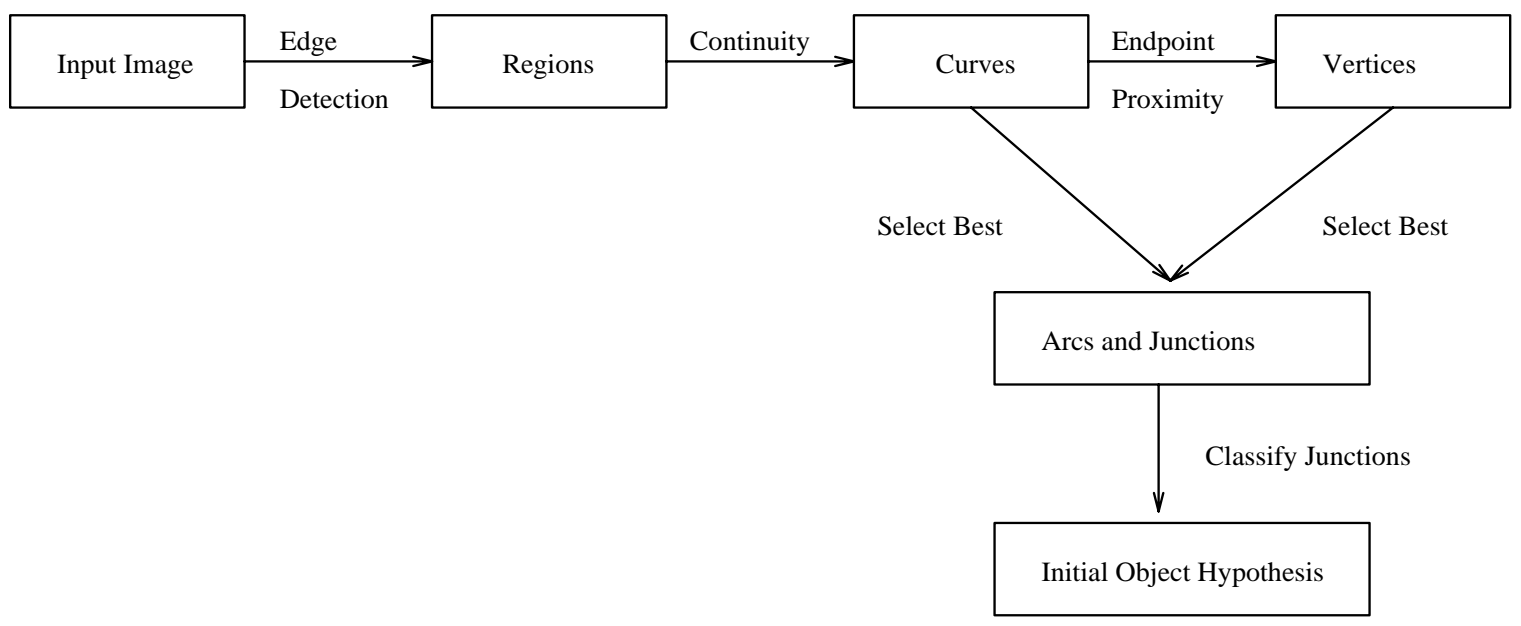

Figure 4: An overview of the initial processing sequence.

we have chosen Malik's line labeling algorithm with some additional restrictions on the object domain.

In the next few sections, we concentrate on the details of the various groups of knowledge sources in the POLL system. These are grouped into the perceptual (or low level) knowledge sources, diagnostic knowledge sources, and the repair knowledge sources, as shown in Figure 2. The low level knowledge sources, which bootstrap the system and form the first hypotheses on the blackboard, will be discussed first.

\section{Obtaining an Initial Line Drawing}

The processing in the POLL system is initiated by feeding it an input image. In order to go from the image to the $3 D$ interpretation, some of the modules need to initially produce intermediate results. Initially, this results in a certain sequence of processing as shown in Figure 4. This sequence is not fixed by the architecture, but is determined by data flow constraints. This is discussed in more detail below in Section 4.1. This processing is used to analyze the input image and create a reasonable approximation of the structure of the image for further analysis. Weak membrane edge detection is performed. The detected edges are grouped using a continuity module, and have vertices assigned at their endpoints. These vertices are collected using spatial proximity to group junctions for line labeling. The junctions are then classed and typed. This gives sufficient information for the first pass of the line labeling algorithm.

Since it is expected that none of the processes utilized in this paper will produce perfect results, a richer description of the image data must be made than what would be necessary for a line drawing. The details are dicussed below. 


\subsection{The Representation of Piecewise Continuous Objects in a Blackboard}

The blackboard database contains a variety of data, including the initial intensity image, the regions, curves, vertices, and object hypotheses that are output by the knowledge sources. To discuss the organization of the blackboard system being used in this research, some vocabulary is needed. The terms that are used here are those used in GBB (Generic Blackboard), the blackboard shell used to implement POLL, and are given in [58]. The term "blackboard" used by GBB will be replaced with "GBB blackboard" so as not to confuse the generic blackboard ideas discussed before with the specific implementation of those ideas in GBB.

A GBB blackboard is a hierarchical tree structure composed of GBB blackboards and spaces. Spaces are used to contain and index units. Units are the basic blackboard objects. When a unit is created it is stored on a space. Such a unit is said to have been instantiated. Spaces are stored, in turn, on a GBB blackboard. Indexing of units on spaces is optional in GBB, but in image processing it can improve the efficiency.

Units hold the hypotheses for entities that are found in the image. For the curvilinear line labeling paradigm, for example, there are units for the arcs and the junctions. Each unit can store three types of information. Slots hold the basic data, such as the name and label of a junction. Indexes determine how the unit will be stored on the space. GBB permits a wide range of indexing possibilities, including Frenet box ranges which are useful for units which are not restricted to a single point. The final type of information is a link. Links relate a given unit to other units. Different types of units may be related using links.

The units used in POLL's blackboard system are as follows:

- Frame: the initial input data before analysis.

- Figure: a group of regions, curves and vertices.

- Region: a two dimensional array of spatially connected pixels

- Curve: a one dimensional array of spatially connected pixels

- Vertex: a point at the end of a curve

All of the units could have been stored on a single space since they are all indexed in the same coordinate system. This would have kept the database overhead to a minimum, but would have made retrieval of a particular unit type more time consuming. By placing each type of unit on a separate space, retrievals can be done more quickly.

Each of the proposed modules uses a subset of these units for its processing. Table 1 summarizes the mapping between modules and blackboard objects. The slots for the units reflect 
the needs of the modules using that unit. Some slots store information that is used by only one module. As an example, the frame unit stores the filename of the input data, and all units have index variables stored. All units have a belief slot which gives a rough interpretation of the importance of this unit at the given point in time. If a hypothesis has belief zero, it is not used in further processing although it remains on the space. Although removing a hypothesis with belief 0 would save storage space and speed retrievals, it would violate one of the design goals by making recovery from mistakes impossible.

The links between units can be divided into four categories. All links are bidirectional, although this is not dictated by GBB but rather by the needs of this application. Adjacency links describe spatial adjacency relationships. An example of an adjacency link is the neighboringcurves link in the curve unit. This link joins two curve units that are spatially adjacent. Adjacency links can also join different unit types. An example of this is the link between regions and their boundary curves. The region side of the link is called bounded-by-curve, and the curve side is called bounds-region. A second type of link also exists between regions and curves. This link is used for curves that are in the interior of regions. Units of the same type are also linked as supporting-hypotheses and competing-hypotheses. The final class of links used are supplementary hypotheses. An example of a supplementary hypothesis is a missing edge that is hypothesized from a line labeling failure.

As we pointed out before, the processing sequence in Figure 4 is not fixed by architecture but is a consequence of the data flow constraints in the processing. Since the knowledge sources are independent, they do not call each other directly. The initial processing is controlled by the blackboard itself. As an example, the creation of a new region triggers the curvilinear grouping module to create the edges. This is not done by the module that created the new region, but is done as a consequence of placing a new region on the blackboard. Similarly, the curvilinear grouping module does not directly call the vertex module. The vertex module is automatically called by the blackboard when the curve unit is instantiated and placed on the blackboard. This type of processing greatly enhances the consistency of the blackboard.

\subsection{Creating the Initial Blackboard}

Since the initial blackboard database is the foundation for all future processing, it is important that it be an accurate representation of the important events in the image plane. It is not necessary that it be perfect. In fact, if it were possible to reliably create a perfect initial blackboard database, the integration that is the subject of this paper would be unnecessary.

During the initial analysis of the input image, competing hypotheses for image events are 
instantiated. These competing hypotheses will make it possible to backtrack and correct poor initial decisions at a later point in time when global constraints are applicable. With this strategy, operations like thresholding that are normally fragile can be used with more confidence.

The first line drawing hypothesis is created in three parts. Section 4.2.1 describes the separation of the frame into coherent regions. In Section 4.2.2, the processing which starts with the region units and creates a competing set of curve and vertex units is described. Once the curve and vertex units have been established, the junctions need to have the number of incident arcs in the initial figure hypothesis identified, and type (i.e. three tangent, curvature-L) determined.

\subsubsection{Finding Regions}

The purpose of the initial processing is to create a reasonable representation of the region, curve and vertex units from the frame unit. All of the processing described in this section is of a local nature. Local processing is advantageous at the early stages because it is potentially massively parallel, although this virtue is not exploited in this implementation. The disadvantage of local processing is that information in a small neighborhood is not always reliable in a more global context. Since it is known that the information may not be globally reliable, alternate hypotheses are kept. This makes it possible for later processing to backtrack and reverse decisions that were good locally, but which fail in a more global setting.

The processing in this section will be demonstrated using the image in Figure 5 . This image was captured in the Pattern Recognition and Image Processing (PRIP) laboratory at the Michigan State University.

The first step in the processing of the real image is to find the edges using the region based edge detection. As was discussed in Section 3.1, the weak membrane model with GNC algorithm is used for edge detection. Although GNC claims to produce thin edges, it did not always in practice. The reason for this failure was that many of the edges in the processed images were ramp edges instead of step edges. This was caused by two factors. The first factor is discretization error. Since the division between regions does not always coincide with the division between pixels, some pixels will contain a mixture of two regions. This produces a narrow ramp edge instead of a crisp step edge. The second reason for GNC to produce thick edges is that some of the objects (bath tub toys) that were imaged do not have flat sides. The sides of the toys that appear to be planar are slightly curved. This curving produces ramp edges instead of the step edges that GNC expects.

GNC responds to the ramp edges as repeated step edges and produces thick edges. The edges found by GNC when run on the image in Figure 5(a) are shown in Figure 5(b). Although 


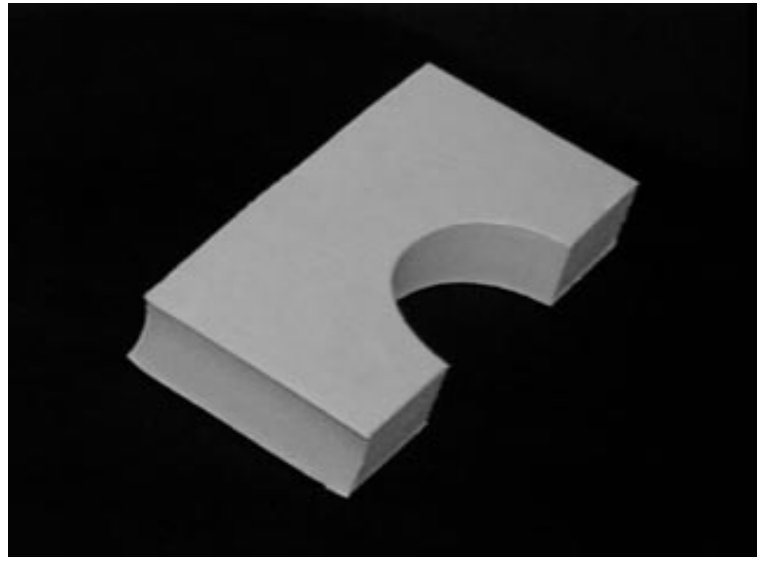

(a)

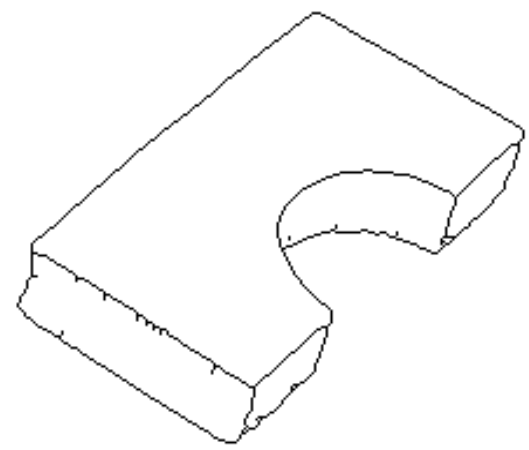

(c)

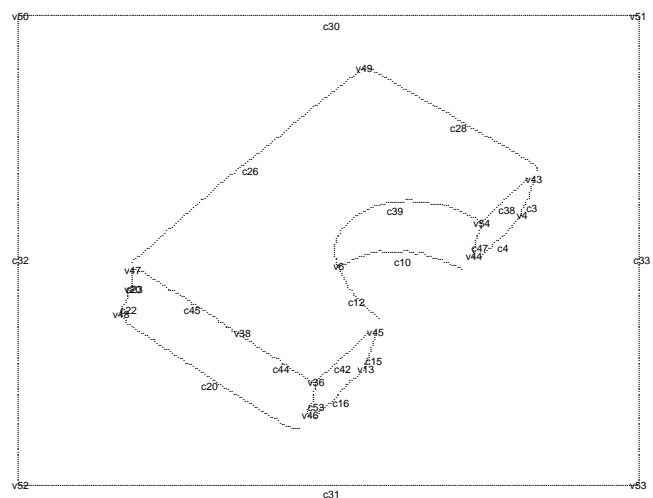

(e)

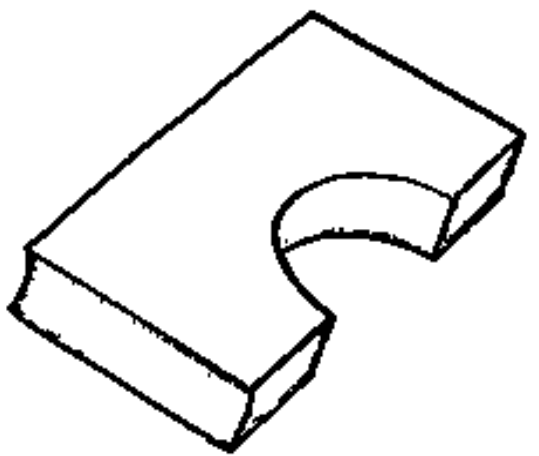

(b)

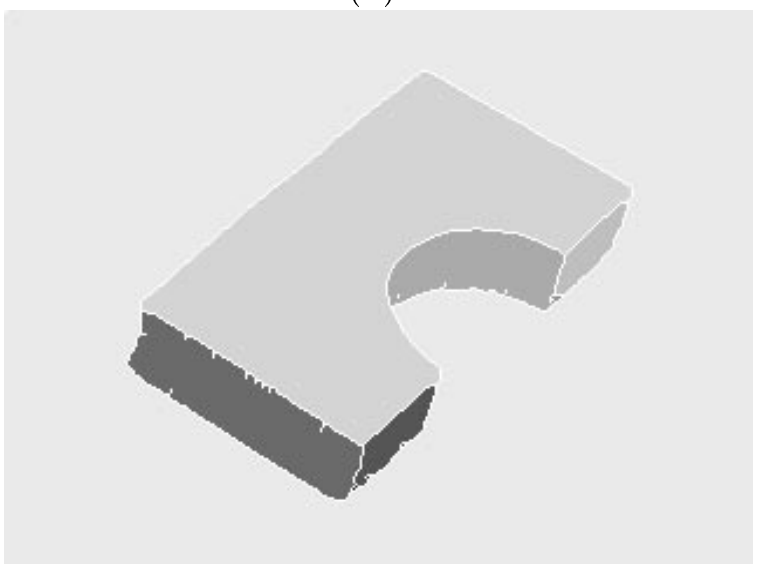

(d)

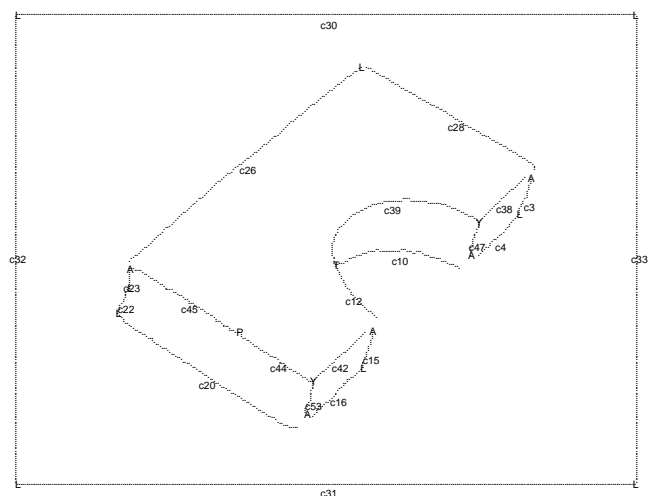

(f)

Figure 5: An example image on which the initial processing is illustrated. (a) The input intensity image. (b) The result of weak membrane edge detection. (c) The thinned edges. (d) The regions identified as connected components. Each connected component is represented as a separate gray shade. (e) The initial curve and vertex units found. (f) The initial vertices typed (A: arrow junction, T: T-junction, Y: Y-junction, L: L-junction, P: Phantom junction). 


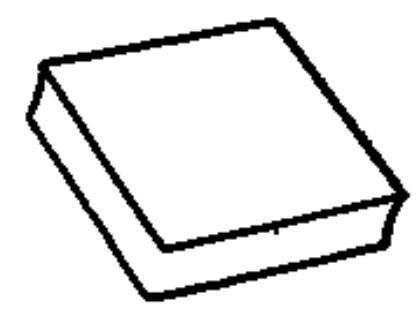

Figure 6: The image shown in Figure 1 after processing with GNC. Notice that the front edge of the cube is missing, as expected.

this image was correctly segmented in terms of object semantics, some images were not. For example, the edges detected in the image in Figure 1 is given in Figure 6 . The front corner edge of the block is not detected, as predicted, because the intensities do not provide evidence for an edge in this location.

In order to create one dimensional curves from the thick edges of GNC, thinning was done. The result of running the thinning algorithm on the example image is shown in Figure 5(c). The thinned edges are the borders of the region units.

To get the region units, a connected components algorithm was run [59], using an eight connected neighborhood relationship. Figure 5(d) shows the connected components found from Figure 5(c). Different grey values correspond to distinct regions. Small regions are given a unit with belief 0 , and then included in the largest neighboring region. A region is labeled as small if it has fewer pixels than the threshold given in Appendix A. Any unit with belief zero is not included in further processing, although it is still available on the blackboard for retrieval should it be needed later.

\subsubsection{The Curves and Vertices}

The boundaries of the region units are used to find the initial curve units. First the complete boundary of each region is given a single curve unit. This curve unit is initially given a belief value of one (the belief values range from one to ten, but the only significance of the belief value is relative to the other competing units of similar type), meaning that it should be included in processing but is not considered to be a favored hypothesis at this point. This boundary is then broken into pieces at any point with more than three edge neighbors (triple points). Each piece is a distinct competing curve unit with a belief value of 2 signifying that these curve hypotheses 
are more perceptually significant than the initial ones and should take precedence in processing. If no competing hypotheses are found, the initial curve hypothesis has its belief raised to 2, meaning that it has gained perceptual significance. The curvilinearity algorithm described in [21] is then run on the triple point curve units, or the original boundary curve unit if it was unbroken by vertices. If corners are found by the continuity algorithm, competing curve units are created for each segment, with a belief value of three. If no corners were found, the previous curve hypothesis has its belief value raised to three. This leaves a unique hypothesis that is thought to be best at each of the points on the boundary of the region. Since the continuity algorithm is consistently finding better hypotheses than what triple points alone could provide, these hypotheses are given the highest confidence of the three curves initially. At the end of each of these curve units, a vertex unit is instantiated.

There are edge points that are found by GNC that do not fall on the boundary of a region. Some of these points are caused by hairs left on the edges by GNC that have been thinned. Some of the other edges on the interior of a region are partially detected edges. To collect these edges into reasonable structures, the edges that are not on the boundary for each region are grouped using the curvilinear grouping algorithm. By grouping only within single detected regions, undesirable groupings between distinct regions are eliminated. These groupings are given a belief value of three if they are more than a few pixels long (the threshold value is given in Appendix A), and a belief value of zero otherwise.

At this point, a set of competing curve units has been created. Each curve unit will have either no endpoints (as with the boundary of an isolated sphere), or two endpoints. Some of the curve units are only a few pixels long, particularly those near the corners of the regions. Competing vertex units have not yet been created. Since the continuity algorithm typically leaves several bends near a vertex, particularly when working with real data, these small curves and vertices need to be combined. The method for doing this initially is simple. When a vertex is created, it tries to join with any other vertex in a local neighborhood to create a stronger vertex. The size of this local neighborhood is determined by a threshold, shown in Appendix A. If a vertex is combined, the old vertex is given a belief zero. An artifact of this vertex combination is that there are short curves with both endpoints at the same combined vertex. These curve units are given a belief zero. This cleans away many short, perceptually unimportant curves.

This processing results in an initial set of vertices and curves that form the first figure hypothesis. The result for the example image in Figure 5(a) is shown in Figure 5(e). The labeling scheme is as follows. Curves are labeled with the letter "c" and an identification number. Vertices are labeled with the letter "v" and an identification number. The label for a 
curve is placed to the lower left of the middle of a curve. The label for a vertex is placed at the vertex. Around the edge of the image is a frame that completes any junctions that reach the edge of the image. This frame will be shown in all future images, and is labeled a priori as a jump edge.

At this point the blackboard contains regions, a group of competing curve units, and a group of competing vertex units. Within the groups of both curve units and vertex units the competing units have been labeled with belief values to determine which appears to be best at this point in time. The blackboard also contains the beginning of the first figure unit: the collection of all non-competing curve units of highest belief value and the collection of all non-competing vertex units of the highest belief value. For the figure unit to be complete the vertices must be classified and typed. These operations are discussed next.

\subsubsection{Identifying Junctions}

First vertex units are classified by the number of incident arcs. This classification yields four categories, junctions with one incoming arc, junctions with two incoming arcs, junctions with three incoming arcs, and junctions with four or more incoming arcs. The domain assumptions made in this work exclude vertices with more than three arcs, so junctions in the fourth category are outside the scope of this paper and can be flagged as erroneous immediately. The domain of piecewise smooth surfaces discussed in [30] does not have this restriction.

A much more challenging problem than classifying the junctions is typing them. A summary of the attributes that determine junction type is given in Table 2. The table does not distinguish between Arrow and Y junctions. This distinction is made by examining the angle between the incoming arcs. If one of the angles is greater than $180^{\circ}$, the junction is an Arrow. If all three angles measure less than $180^{\circ}$ the junction is a $\mathrm{Y}$.

In order to assign a type to the junctions, each of the curve units must have a tangent and curvature value at both endpoints. Finding curvature is a difficult problem. Therefore the curvature values that are calculated should be treated with suspicion. Estimating tangent direction is somewhat easier. Since later processing can help to correct mistakes, it is not essential that the curvatures and tangents be perfect. Curvature and tangent direction estimations are regularized using the continuity module to preprocess all curves. This module smooths the curves, removes outliers, and separates the curve at discontinuities. Without this preprocessing, the estimations would be more unreliable.

The simplest way to find curvature is to fit a circle to a set of points, and find the radius and the center. The multiplicative inverse of the radius is the curvature. A normal to the fit 
Table 1: The mapping between modules and blackboard units.

\begin{tabular}{|l|c|}
\hline Module & Unit \\
\hline \hline Curvilinearity & curves \\
\hline \multirow{2}{*}{ Symmetry } & regions \\
\cline { 2 - 2 } & curves \\
\hline \multirow{2}{*}{ Proximity } & curves \\
\cline { 2 - 2 } & vertices \\
\hline \multirow{2}{*}{ Edge Detection } & frame \\
\cline { 2 - 2 } & regions \\
\hline \multirow{2}{*}{ Line Labeling } & figure \\
\cline { 2 - 2 } & curves \\
\cline { 2 - 2 } & vertices \\
\hline
\end{tabular}

Table 2: A summary of the tangent and curvature properties of junctions. Arrow and Y junctions $\left(^{*}\right)$ are distinguished on another basis. The symbol given will be used to represent this type of junction in later figures.

\begin{tabular}{|l|c|c|r|r|}
\hline Class & Type & Symbol & Tangents & Curvatures \\
\hline \hline \multirow{2}{*}{ One arc } & Terminal & TM & None & None \\
\hline \hline \multirow{3}{*}{ Two arcs } & Curvature L & CL & Equal & Unequal \\
\cline { 2 - 5 } & $\mathrm{L}$ & $\mathrm{L}$ & Unequal & Doesn't Matter \\
\cline { 2 - 5 } & Phantom & $\mathrm{P}$ & Equal & Equal \\
\hline \hline \multirow{3}{*}{ Three arcs } & Arrow $(*)$ & $\mathrm{A}$ & Unequal & Doesn't Matter \\
\cline { 2 - 5 } & $\mathrm{Y}(*)$ & $\mathrm{Y}$ & Unequal & Doesn't Matter \\
\cline { 2 - 5 } & $\mathrm{T}$ & $\mathrm{T}$ & Exactly Two Equal & Same two Equal \\
\cline { 2 - 5 } & Three Tangent & $3 \mathrm{~T}$ & All Equal & Two Equal \\
\hline
\end{tabular}


circle at the endpoint can be found from the vector starting at the center of the circle and ending at the endpoint of the arc. The tangent direction is perpendicular to the normal. The fitting is done using a nonlinear least squares method found in [60]. Circular arcs were fit in a neighborhood of the endpoint, with the size of the neighborhood determined by a parameter given in Appendix A.

Although a straight line can be thought of as a circle of infinite radius, this is numerically troublesome. To avoid this problem, straight lines are fit in a neighborhood of the endpoint. The size of this neighborhood is determined by a parameter given in Appendix A. The curvature for a straight line is zero, and the tangent direction is calculated from the slope. The squared error, calculated in units of pixels squared, between the circular fit and the linear fit is then compared and the model with the better fit is accepted. The curvature values calculated in this fashion are rough estimates. Therefore comparisons like those suggested in Table 2 must be revised. Even with synthetic data, it is unlikely that two curvatures or tangents would be exactly equal. To compare tangent directions, a threshold was used on the difference. This threshold is given in Appendix A. This threshold was set arbitrarily, but is not critical to the performance of the system since later processing can change this threshold to correct mistakes.

The curvature values were found to be very unreliable, particularly in the real images that were used. There are several reasons for this. The objects used in the images in this paper have circular arcs, which project to ellipses. Fitting a circle to an ellipse, particularly at the ends of the major axis where the curvature is changing rapidly is difficult. Another problem is that the continuity algorithm may not break the upper and lower halves of an ellipse exactly at the corner. This means that the curvature will be calculated starting at different places on the ellipse, which will certainly lead to different curvatures. To get around this problem, curvatures were placed into only three classes, which are described in Appendix A. Curvatures will be considered to be equivalent if they fall into the same class. It would have been possible to fit an ellipse to the curve data instead of a circle, but this is computationally more expensive and unnecessary.

With the curvature and tangent information, and suitable definitions of equivalence among tangents and curvatures, the classified vertices in the initial figure unit can be typed. This information completes the initial figure unit, that is the first hypothesis for the arcs and junctions in the imaged scene.

Although errors in the initial processing can be repaired later, it is important that the first figure unit be an accurate representation of most of the important events in the image plane. Perfect line drawings should not be expected. Figure 7 shows some other example images with 


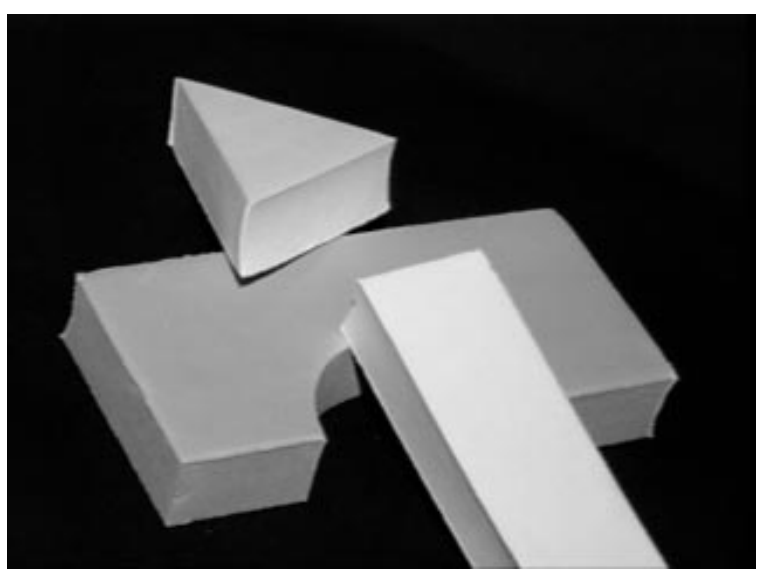

(a)

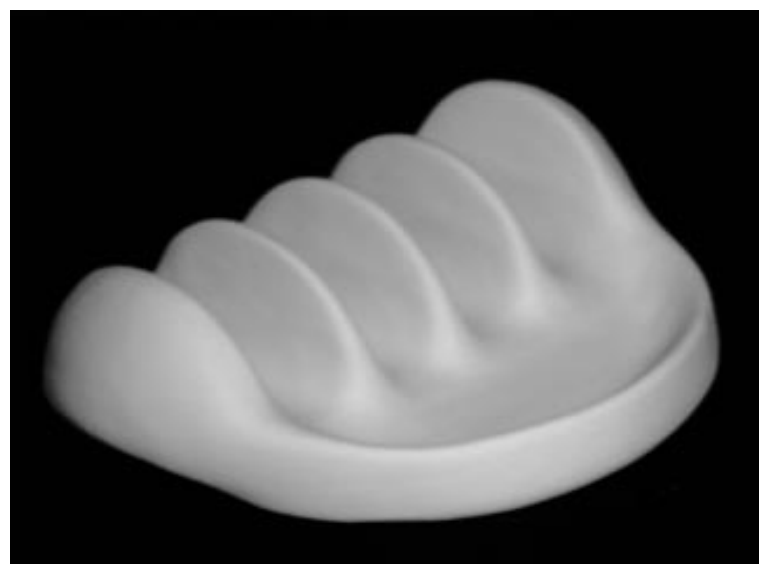

(c)

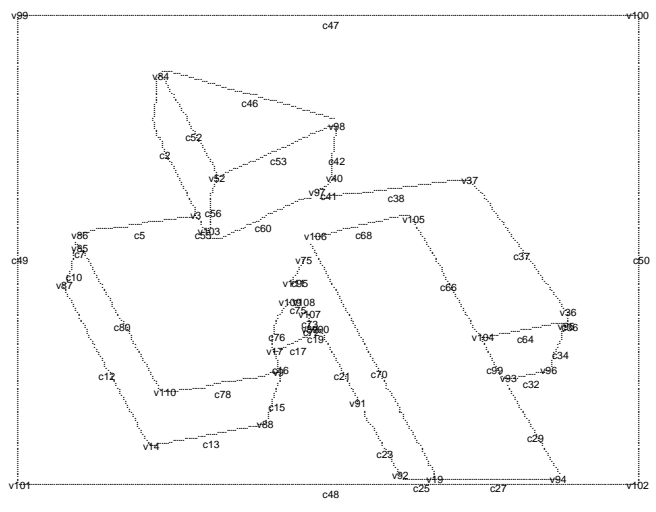

(b)

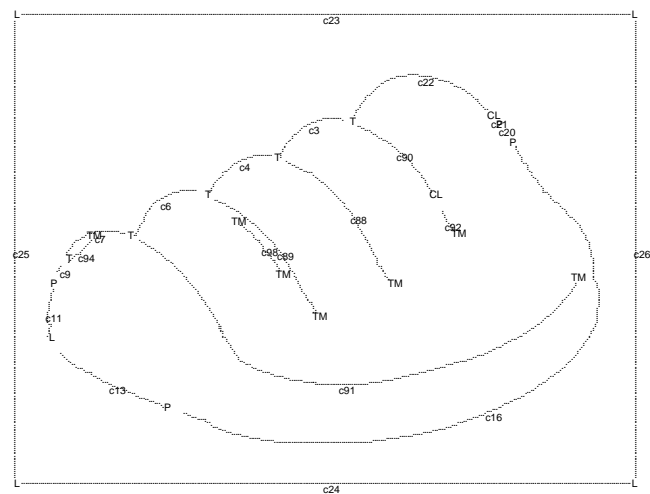

(d)

Figure 7: (a) The input image of a set of blocks. (b) The first figure unit computed from (a). (c) The input image of a taco holder. (d) The first figure unit computed from (c).

the initial figure hypothesis results.

\section{$5 \quad$ Generating Line Labeling Diagnostics}

Line labeling has long interested vision researchers because it provides a way to use local information about curves and vertices to build a more global interpretation of image data. The practical difficulty in using line labeling, and especially in curvilinear line labeling, is that the local information about curves and vertices is often imperfect. Since the rigorous mathematical framework built in the proof of the junction catalog assumes that a perfect line drawing is available as input, the algorithm is doomed to failure in all but the most contrived cases. This is particularly true since the algorithm was not designed to provide diagnostic information about the nature of the failure. In this section, a revised line labeling algorithm will be presented that 
can provide some diagnostic information about line labeling failures.

\subsection{Detection of Line Drawing Problems}

The algorithms that have been used in Section 4 to extract line drawings from images cannot produce perfect output. Therefore it is important to be able to analyze a line drawing and evaluate its strengths and weaknesses. This section will show a modification to the standard line labeling scheme proposed by Malik [30] which provides better diagnostic information about the weaknesses of the current line drawings. This diagnostic information will be entered into the blackboard database and later be used by other modules to fix problems in the line drawing. The line labels used for the domain of piecewise smooth objects are the following:

$\rightarrow \rightarrow$ A limb edge is a depth discontinuity that is formed when the line of sight is tangent to the surface and the surface occludes itself with respect to the viewpoint. The direction of the arrow is selected such that the surface closest to the viewer is on the right hand side of the arrow as it is followed from tail to head.

$\rightarrow$ A jump edge which is a depth discontinuity which is not a limb edge.

+ A convex edge where the visible angle between the surfaces is greater than $180^{\circ}$.

- A concave edge where the visible angle between the surfaces is smaller than $180^{\circ}$.

In the piecewise smooth object domain, the junction catalog is guaranteed to give one or more correct labelings for each object in the object domain. An object that is not within this domain may still be legally, and perhaps even correctly, labeled. To restate this in a way that is more relevant to this discussion: there is no guarantee that if a mistake is made in the initial line drawing that line labeling will fail to interpret the image. Therefore, line labeling cannot be the only arbiter of correctness in line drawings. However, when possible, it can contribute towards this goal. This section will demonstrate how line labeling can be instrumental in fixing incorrect line drawings.

One of the difficulties with using line labeling as a diagnostic tool is that there are often multiple interpretations of a single object. To reduce the combinatorics of the line labeling, the POLL system uses a heuristic called the floating object heuristic to initiate labeling of the line drawings. We assume that the image has been segmented into regions. Further, we assume that these regions have been correctly labeled as either background or foreground. The objects of interest should be in the foreground. The labeling of the background region is a method for performing figure-ground separation, which is one of the Gestalt principles. This figure-ground 
separation is done by hand in the POLL system, although it would be preferable to have the system select the background automatically. Using this terminology, the heuristic is defined as follows:

Definition 1 Floating Object Heuristic: All outside arcs (that is, those that are adjacent to the background region) in a line labeling should be labeled as either limb or jump edges with the direction being such that the foreground region will be interpreted as being in front of the background region. The labels on inside arcs are not restricted.

There are times when the floating object heuristic won't work, such as when part of an object is being viewed through a hole. A similar heuristic was used before by Winston [61]. Line labeling with the floating object heuristic is much less combinatorially explosive than ordinary line labeling. There are two contributing factors. The first factor is that there are fewer interpretations of outside junctions. A casual inspection of Malik's original junction catalog, shows that most of the legal interpretations for inside junctions will no longer be legal interpretations for outside junctions thus reducing the number of possibilities. A second factor that improves the combinatorics of line labeling is that phantom junctions are not necessary with this heuristic, since a necessary phantom junction can be identified by the line labeling process.

If an image can be segmented into foreground and background regions, outside junctions that have been labeled by the floating object heuristic will have at most two interpretations, except for $\mathrm{T}$ junctions which may have as many as four interpretations. This can be verified by a case analysis on the junction catalogue and a counting argument.

Table 3 shows a vast improvement in combinatorics for outside junctions with the floating object heuristic. Five different outside junctions have a unique labeling with the floating object heuristic. These unique labelings of arcs on a local basis will improve the combinatorics of labeling inside arcs as well. This combinatoric improvement can also be exploited to improve the line labeling paradigm. Outside arcs and outside junctions are providing the strongest constraints. When this information is used first in the line labeling algorithm, the legal labelings on the inside arcs are also restricted. Algorithm 1 is the modified line labeling algorithm which takes advantage of this combinatoric reduction and which provides diagnostic feedback.

The principal advantage of Algorithm 1 over the three step algorithm proposed by Malik in [30] is that diagnostic information is provided for the blackboard in the form of the failed vertex. Since the number of legal line labelings is often reduced to a single set by the improved combinatorics, this diagnostic information can pinpoint the area of the line drawing that is incorrect. This makes it possible to make corrections. Figure 9 shows the curves and vertices identified for the image in Figure 1. When the modified line labeling algorithm is run on this 
Table 3: The number of legal labelings for outside arcs.

\begin{tabular}{|l|c|c|}
\hline Junction & Inside Labelings & Outside Labelings \\
\hline $\mathrm{TM}$ & 1 & 0 \\
\hline $\mathrm{L}$ & 6 & 1 \\
\hline $\mathrm{CL}$ & 4 & 2 \\
\hline $3 \mathrm{~T}$ & 2 & 1 \\
\hline $\mathrm{A}$ & 3 & 1 \\
\hline $\mathrm{Y}$ & 5 & 1 \\
\hline $\mathrm{T}$ & 12 & 4 \\
\hline
\end{tabular}

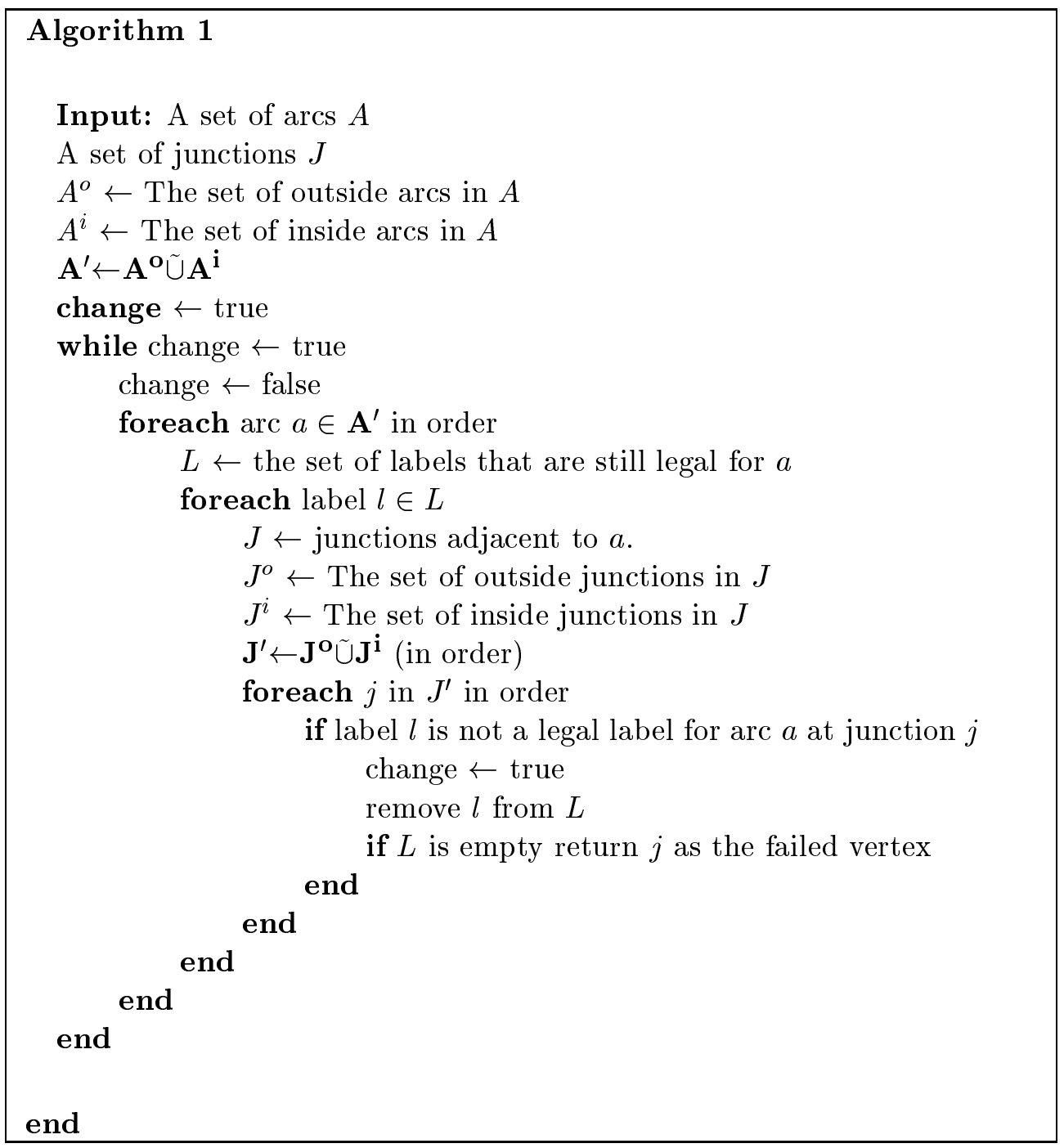

Figure 8: The revised line labeling algorithm. The notation $\tilde{U}$ is used to indicate that the sets of junctions and arcs are concatenated, while maintaining the order. 


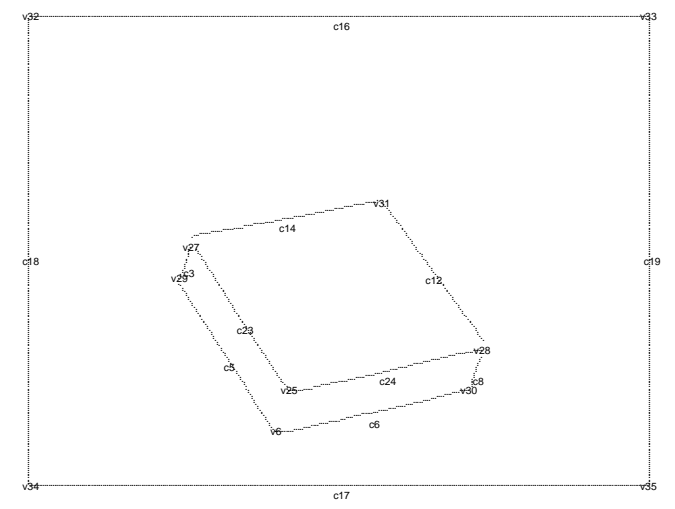

Figure 9: The junctions and curves found from processing Figure 1.

intermediate result, the junction "v25" is correctly diagnosed as the location of the problem. An assessment of the results and more examples are shown later in Section 7.

Figures 10-11 give more examples of the results obtained by using the diagnostics coming from the revised line labeling algorithm. In these examples, the vertices that were flagged as being the cause of the labeling problems were indeed the troublemakers. This localization of the problems will give the repair knwoledge sources a starting point for forming hypotheses.

\subsection{Common Problems with Line Drawings}

When line drawings are obtained from real images, there are a number of problems that can occur. In this section, line labeling problems that result from the processing described in Section 4 will be discussed. These problem line drawings will be analyzed using the floating object heuristic, and the resulting diagnostic information will be discussed. Since the line labeling paradigm has no knowledge of the inner workings of the other modules, it is not able to fix any of the problems that it detects without violating the independence of knowledge sources. The suggestions for fixing the problems will come from control knowledge sources to be discussed in Section 6 , and the correction modules.

In the example images that were used in testing this system there were four common problems: missing or incomplete edges, mislabeled junctions, missing junctions, and extraneous junctions. There are other problems that can occur in line drawings, such as extraneous edges, but the vast majority of the incorrect line drawings that were used to test this system fell into these four categories. This is a result of a conscious decision to select parameters for the weak membrane edge detection that undersegment the image, i.e. that divide the image into too few regions. 


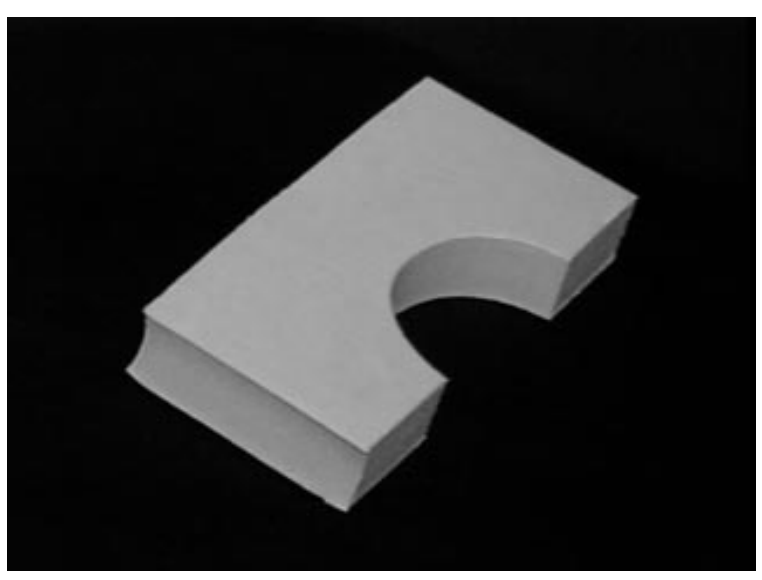

(a)

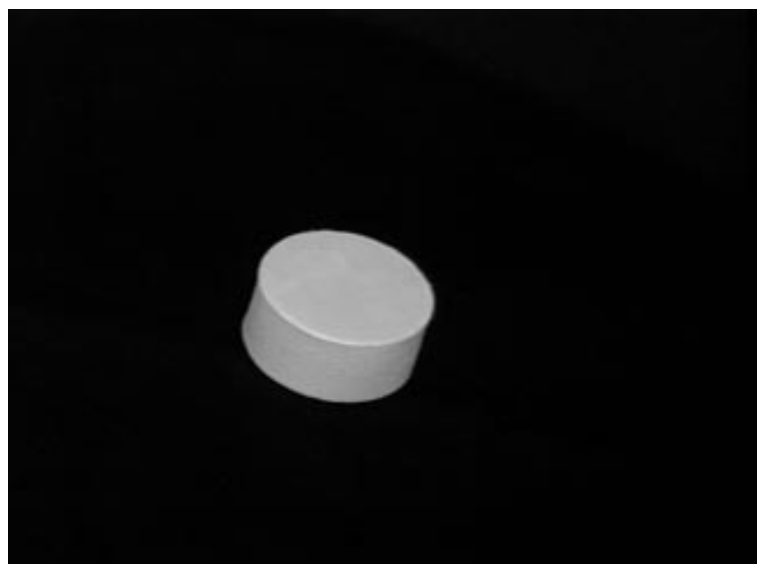

(c)

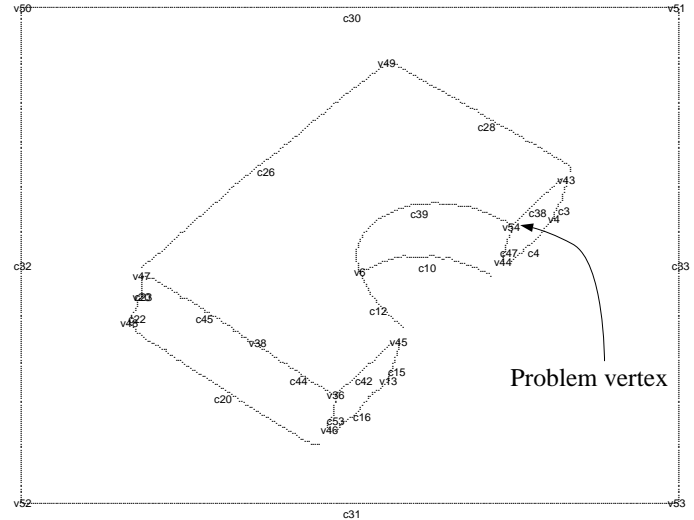

(b)

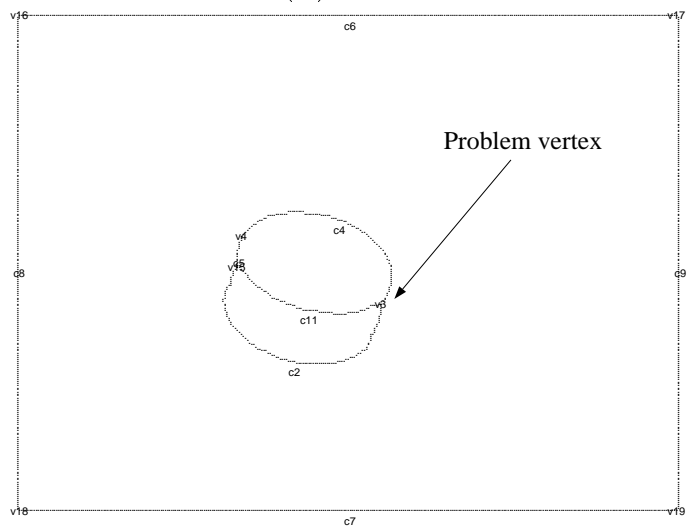

(d)

Figure 10: Some example results of diagnosis. (a) The input image of Figure 5. (b) The vertex "v54" is diagnosed as problematic. The reason is that there should be a phantom vertex along the curve "c39" adjacent to vertex "v54." (c) The image of a right circular cylinder. This is one example where the ability to distinguish between Arrow junctions and Curvature L junctions is crucial for the line labeling algorithm to work properly. (d) The vertex "v3" is flagged as the problem location. 


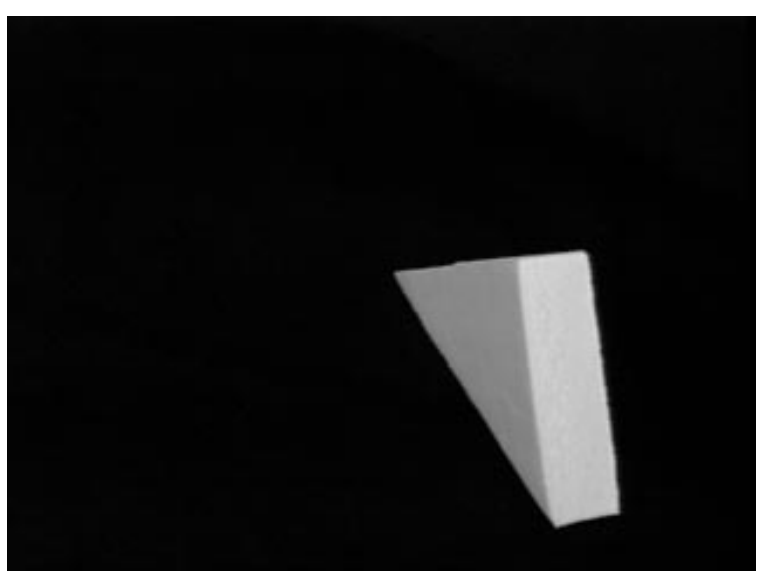

(a)

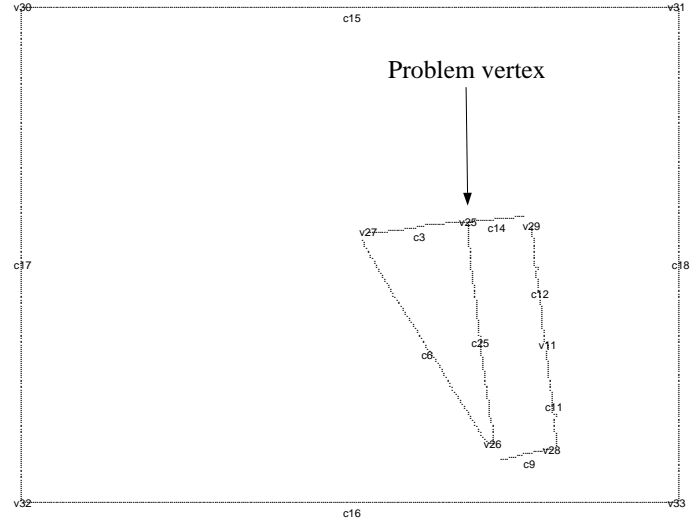

(b)

Figure 11: Some more example results of diagnosis. (a) The image of a triangular prism from an accidental viewpoint in which an arrow junction appears lika a $\mathrm{T}$ junction. (b) The diagnostic module flags correctly vertex "v25" (the erroneous T junction) as the problem location.

Incomplete and missing edges occur when the edge detection algorithm does not completely separate two regions. An example of an unavoidable missing edge was shown in Figure 1. Mislabeled junctions occur in line drawings because it is difficult to find the type of a junction using only local information. Confusion occurs between junction types of the same class, for example three tangent, arrow, $\mathrm{Y}$, and $\mathrm{T}$ vertices. These mistakes are inevitable, since in the mathematically rigorous curvilinear line labeling paradigm the only distinction between a $\mathrm{T}$ junction and a $\mathrm{Y}$ is $\mathrm{T}$ junctions have two arcs that have perfectly aligned tangent directions. Since this perfect alignment rarely happens in practice due to positional noise, a threshold was used to determine how close to perfect alignment two arcs must be to be considered a $\mathrm{T}$ junction. When this is done, a Y junction or an arrow junction that falls within this thresholded range will be improperly typed as a $\mathrm{T}$ junction.

The most difficult junctions to properly analyze are the curvature-L junctions and three tangent junctions. In a local neighborhood, they are very similar to arrow junctions and L junctions, respectively.

Missing junctions are another problem in the input line drawings. Missing junctions occur when the continuity algorithm does not detect a corner. The only type of junctions that can be missed are L and Curvature-L junctions. Junctions with three incident arcs cannot be missed because they occur at a position where three surfaces are joined, and all such points are forced to be vertices. Junctions with three incident arcs may be mislabeled, or misplaced, but they will not be missing. 


\section{Utilizing Line Labeling Diagnostics for Repair}

Previous sections have described how an initial line drawing can be found from a single intensity image, and how problems in this line drawing can be diagnosed. This section will focus on the repair knowledge sources that were created to use the diagnostic information coming from the modified line labeling algorithm to fix some of the problems in the line labeling, and the controller knowledge source that governs which repair knowledge source is chosen.

This is the point at which the independence of the knowledge sources starts to pay dividends. While it would be possible for the line labeling module and try to correct problems that are the result of other modules, the complexity of this structure would be high. The line labeling module would have to know about all of the data structures from all of the other modules, and be able to modify its data structure to call them.

A blackboard system solves this problem by having each module translate to and from the standard blackboard objects. Control knowledge sources are designed to use the standard blackboard objects alone. Control knowledge sources have no knowledge of the detailed inner workings of the other modules. They only know what blackboard objects are legal input to what modules, and the control parameters, (such as the parameters for edge detection), that each knowledge source has made available to the blackboard. This division of labor makes it possible to make changes within a single module without propagating them into other modules. The control knowledge sources do need to know how to call individual modules, but they do not need to know anything about the internal data structure of the modules. This structure is ideal for experimentation with controlling the modules, since the modules themselves do not have to be altered.

In order to fix line drawings, two types of knowledge sources are needed. The first type are repair knowledge sources. These knowledge sources know how to fix a particular problem in a line labeling. For example, there are repair knowledge sources that can insert phantom vertices into a line drawing, and hypothesize where edges that are missing could be placed. These knowledge sources are instantiated by the controller knowledge source, which has a strategy for selecting which of the repair knowledge sources should be selected next.

We now discuss the knowledge sources that try to correct problems in the partially derived line drawings as a result of diagnostics generated from applying the line labeling constraints.

\subsection{Correcting Errors in Line Labeling}

A variety of problems with the initial line labelings were found by the modified line labeling module. These problems now need to be identified and corrected. There were four modules used 


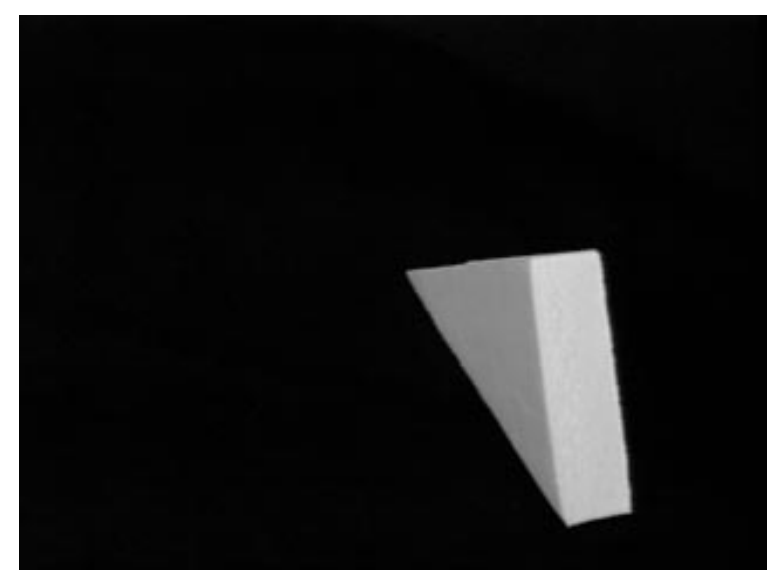

(a)

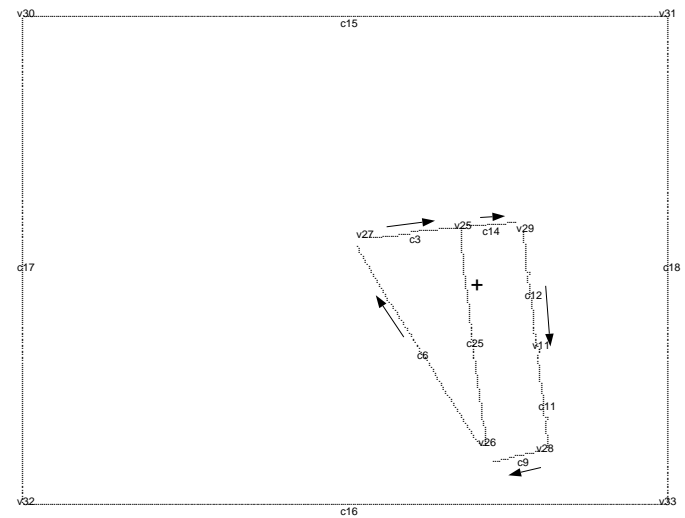

(b)

Figure 12: (a) The input image. (b) The vertex "v25" is corrected from a T junction to an Arrow junction and the figure is relabeled. The labeled arcs and junctions

to create the line drawing: edge detection, curvilinear grouping, vertex proximity grouping, and junction classing and typing. Each of these modules can, and does, make errors. These errors are cataloged in Table 4 . The errors are not mutually exclusive, for example a missing edge will cause two junctions to be of the wrong class.

These errors will be fixed with a set of knowledge sources called the repair knowledge sources. Each repair knowledge source is an expert at fixing one type of problem that can occur in an image. The repair knowledge sources will be activated by the controller knowledge source that decides which of the repair knowledge sources is most likely to fix the current problem vertex and instantiates the knowledge source. The success or failure of the knowledge source will be reported back to the controller, which can then either accept the solution, make more modifications, or stop the processing.

\section{Mislabeled Vertices}

When a $\mathrm{T}$ junction is suspected of causing the line labeling problem one way to reexamine it is to adjust the tangent threshold that was used to label the vertex. In fact, since it is known that the $\mathrm{T}$ labeling is not correct, it is best to set this threshold to zero. If this setting were made initially, $\mathrm{T}$ junctions would almost always be mislabeled. After this adjustment is made, the line labeling is applied once more by the appropriate knowledge source to the new line drawing with the modified junction type. Figure 12 shows the example in Figure 11 where a mislabeled vertex is identified as the problem by the diagnostic module. Figure 12(b) shows the result of applying the repair knowledge sources and the final labeling obtained for this image. 


\section{Missing Arcs}

Since the modified line labeling algorithm can detect missing arcs, a procedure for adding arcs is needed. The problem is approached by hypothesizing where a missing edge could go and then picking the best of these hypotheses. This approach will be useful in cases like that shown in Figure 1 where edge detection of any sort is unlikely to find an appropriate edge.

Assume that the control knowledge source has decided to hypothesize a missing arc. A vertex needs to be found as for the second endpoint for the missing arc. This vertex must be in a region that is adjacent to the problem vertex. The second type of vertex to be considered is a vertex with two incoming arcs. Adding a third arc to this type of vertex would permit an interpretation that is legal in the current object domain. Phantom vertices will not be used for the second vertex since the phantom vertices that are presently in the line drawing are there as the result of failures in the continuity algorithm and have no perceptual significance.

Once the set of second vertex candidates is found, a curve is hypothesized between the first vertex and each of the second vertex candidates. These curves are added to the initial object hypothesis individually, and this new hypothesis has its junctions typed and labeled. If the labeling does not fail at the either of the vertices that were modified, then the new object is put on a list of candidate objects. The best object hypothesis will be chosen from these candidates.

Vertices which already have three incoming arcs could also be used for the second endpoints. This was not done in our case because the object domain specifically excludes objects with more than three faces adjacent to a vertex. If this restriction were to be removed, vertices with three incoming arcs could be given a fourth arc. In this case, it would be best to add a fourth incoming arc to a vertex only if there were no other legal interpretations. This could be regarded as a simplicity criterion, similar to Malik's requirement that a junction with more than four incoming arcs be interpreted in such a way that the minimum number of hidden surfaces are used in the interpretation.

Figure 13 shows the example in Figure 1 which has the front edge missing. We also show in Figure 13 the various arc addition hypotheses actually tried by the POLL system and the final result produced by the repair process.

\section{Adding Phantom Vertices}

There are two important differences between polyhedral line labeling and curvilinear line labeling. The first is that polyhedral line labeling can be aided by linear algebra as shown by Sugihara in [32]. Curvilinear line labeling cannot rely on similar help because, in general, curved arcs cannot be represented adequately with linear equations. The second important difference 


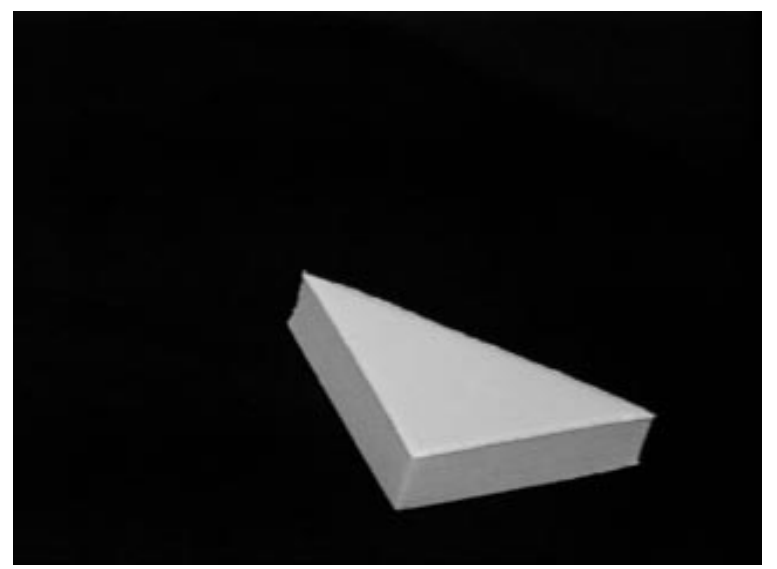

(a)

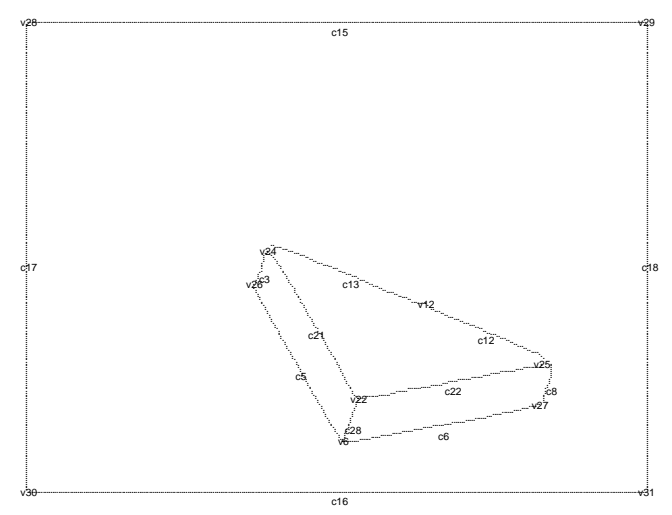

(c)

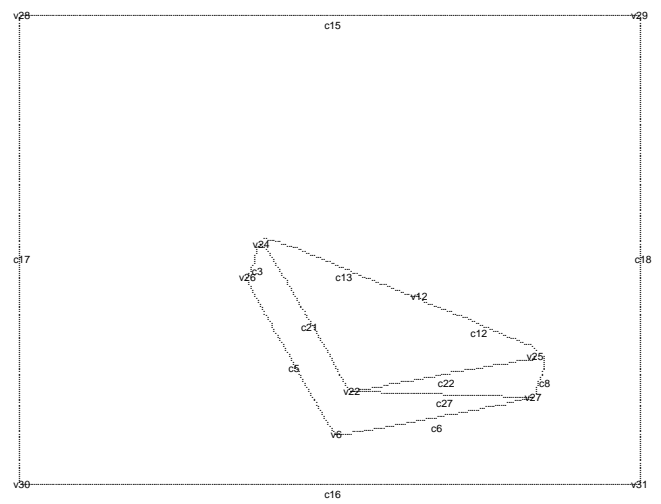

(b)

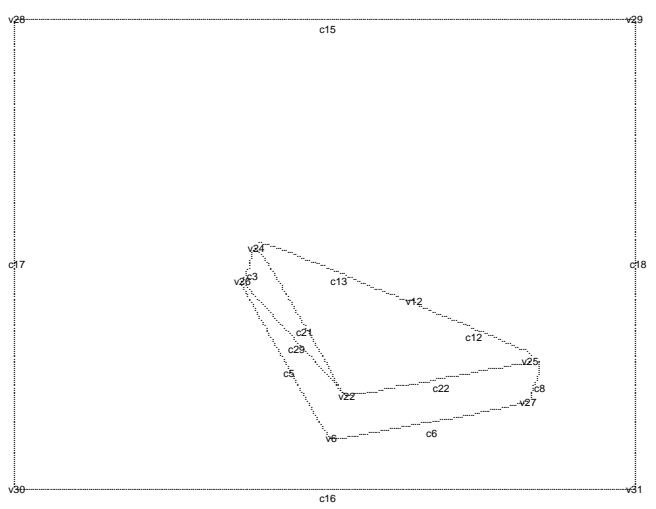

(d)

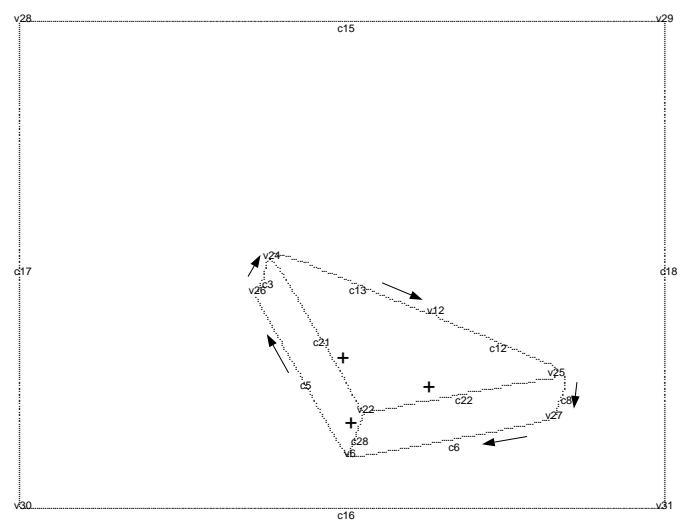

(e)

Figure 13: (a) The input image. (b), (c), (d) the hypotheses generated by the POLL system as possible completion arcs. (e) The final result of repair and labeling. arcs and junctions 


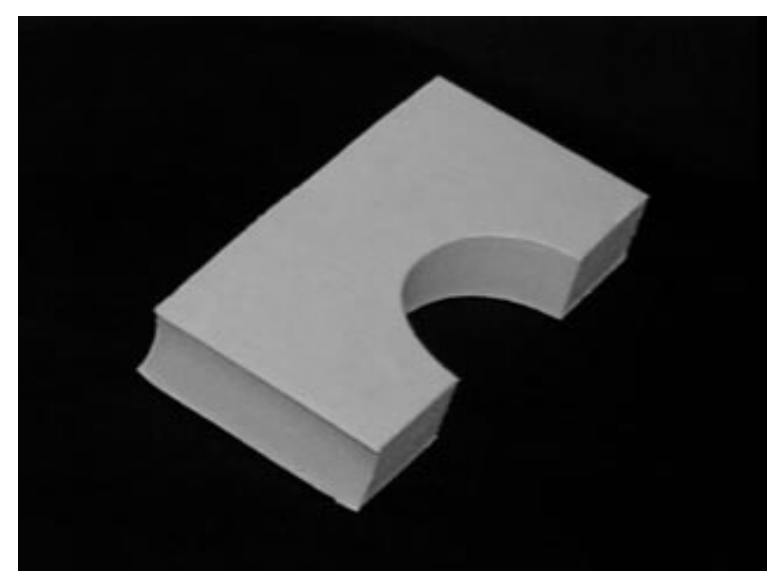

(a)

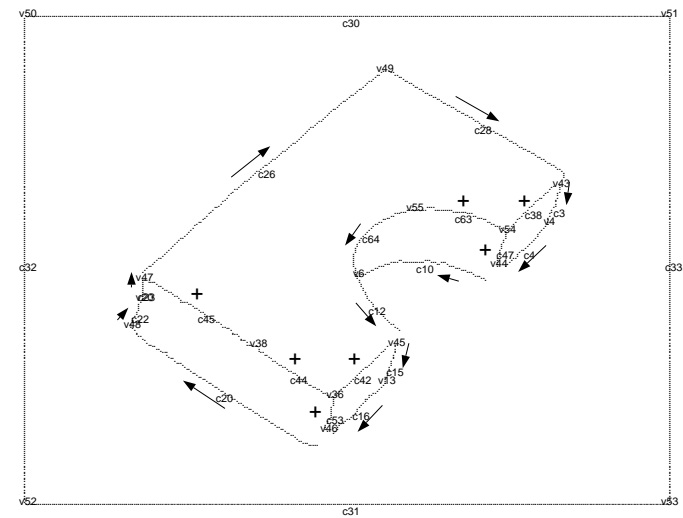

(b)

Figure 14: (a) The input image. (b) The result of repair (adding the phantom vertex "v55") and relabeling.

is that curvilinear line labeling requires all curved arcs to include an extra phantom vertex to accommodate possible hidden limb edges. These extra vertices damage the combinatorics of curvilinear line labeling, and are often unnecessary. It was discussed in Section 5 that the modified line labeling paradigm can flag and locate vertices near a necessary phantom vertex that is missing. Phantom vertices identified in this manner can be inserted into the line drawing and the line drawing relabeled. In this way, phantom vertices do not have to be placed around the line drawing but only put in when and where they are needed.

In order to decide which arcs adjacent to the a problem vertex should receive a phantom vertex, a distinction must be made between curved arcs and linear ones. This distinction was made by comparing a line drawn between the vertices to the actual arc. If the distance between the line and the arc was more than a few pixels (threshold given in Appendix A), the arc was labeled as curved. The threshold is kept small so that if an error occurred it would be more likely to produce an extraneous phantom vertex than neglect a necessary one. All curved arcs adjacent to the problem vertex have junctions added initially since there is no way to diagnose which of the curved arcs needs the phantom vertex. This should still add far fewer phantom vertices to the line drawing than the original strategy proposed by Malik where a phantom vertex was added initially on every curved arc in the image.

Figure 14 shows an example (same one as in Figure 10(a)) where the addition of a phantom vertex has corrected the problem with the line drawing. It also gives the final line labeling generated by the POLL system. 


\section{Other Line Labeling Improvements}

There are other improvements that can be made to the line labeling that have not been implemented. The first improvement is to remove unnecessary phantom junctions from the line labeling. Unnecessary phantom junctions occur in images when the continuity algorithm makes an extraneous break in an edge. This happens most frequently when the edge is noisy. When these unnecessary breaks have their tangents and curvatures calculated and are labeled, they are frequently labeled as phantom junctions signifying that they have no significant difference in either tangent or curvature. The removal of these breaks can be accomplished by hypothesizing a possible grouping and fitting an energy minimizing curve without breaks to get its proper shape. This will produce more reliable curvature values and tangent directions. When the continuity algorithm has finished smoothing the curve, the new curve will replace the old curves in a new figure hypothesis, which will be relabeled to verify the solution.

\subsection{Control of Strategies}

The repair knowledge sources are each capable of using their specialized knowledge to correct exactly one type of problem in an image. Selecting which repair knowledge source should be used first is the job of the controller knowledge source. The strategy implemented here is to use a fixed ordering of the repair knowledge sources. A more elaborate strategy will be necessary as the number of repair knowledge sources is expanded.

The general strategy for fixing these problems is to address only one problem junction at a time. If there are several problems in the image, they will be solved sequentially. A problem is identified as being fixed by a repair knowledge source when the vertex where the failure occurs moves to a different location. A list of vertices where corrections have already occurred should be kept to prevent endless, unproductive repetitions of processing. If a vertex is flagged as being a failure a second time, then the processing should either stop or backtrack to the first correction of the vertex, and try a different correction. This is a search process and, like all search processes, it could be computationally expensive. The more closely the repair knowledge sources can be matched with the diagnosed vertex problems, the less risk of combinatoric explosion.

The following repair knowledge sources are available:

1. Re-type junctions

2. Replace missing arcs

3. Insert a phantom vertex 


\section{Break a vertex into two vertices}

5. Join two close vertices

\section{Remove a phantom junction}

There are two criteria for selecting the ordering of repair knowledge sources. Repair knowledge sources that can be uniquely identified, and are unlikely to be falsely triggered should be used first. Repair knowledge sources that are risky, meaning that they could produce a correct line labeling without correcting the real mistake(s) in the image, should be tried last.

An example of a repair knowledge source that can be uniquely identified is the repair knowledge source that breaks single vertices apart into two adjacent vertices. It can be uniquely identified by the presence of a junction with more than three incident arcs. Similarly the repair knowledge source that groups proximate vertices together is well identified by the presence of a curve incident to one of the junctions that is much shorter than the other incident curves. These knowledge sources should be tried first.

Risk is another consideration when ordering the repair knowledge sources. A repair knowledge source is risky if it can make alterations that may produce a legal line labeling without fixing the underlying problem in the image. In some sense, all of the repair knowledge sources are risky, but two of them are particularly so: the repair knowledge source that inserts phantom vertices and the repair knowledge source that replaces missing arcs. These strategies should be tried as a last resort. Whether phantom vertices damage the combinatorics of line labeling has not been established, but they certainly do not improve the combinatorics and are risky in the sense that they relax an important constraint: that an arc may have one and only one label. The risk in the knowledge source which inserts missing arcs can be limited by checking the arc that gets inserted against the raw image data, to look for a subtle edge that the edge detection algorithm may have missed, or using other constraints such as symmetry.

The repair knowledge source that re-types junctions is neither unique nor risky. It should therefore be tried after the repair knowledge sources that break apart a vertex and joins close vertices and before the repair knowledge source that inserts phantom junctions and missing edges.

The only remaining repair knowledge source is the phantom vertex remover. Since it will not be triggered falsely, it has no risk and can be run at anytime. In the interest of improving the line drawing, it should be run first.

This gives the ordering used for triggering repair knowledge sources in the controller.

1. Remove a phantom vertex 
2. Break a vertex into two vertices

3. Join two close vertices

4. Re-type junctions

5. Insert a phantom vertex

6. Replace missing arcs

As the number of implemented repair knowledge sources grows, more subtle strategies can be developed for selecting among the choices. If a test set of images with ground truth is available, an analysis could be done of the probability of each repair knowledge source fixing a problem for each junction type. This type of experimentation could lead to a better understanding of the frequency of each type of error and more sophisticated control strategies. The development of insight into productive strategies was one of the purposes for using the blackboard paradigm.

\section{$7 \quad$ Experimental Results}

To test the system, the algorithms were run on the test bed of images shown in Appendix B. We have assessed the performance of each part of the system (initial figure units, line labeling and diagnostics, and line labeling corrections) separately and each of these will be discussed in this section.

The results of the performance of initial figure hypothesis modules are summarized in Table 5 . Each of these mistakes is attributed to the module that caused the initial error. As an example, an arrow junction might be missing an arc causing it to be seen as an $\mathrm{L}$ junction. This error would be classified as as missing edge and not a junction type failure since the junction classification is correct given the incorrect input. This system avoids double counting of errors.

There are eight different mistakes that can be made by these four modules. There are two errors that can occur in the weak membrane edge detection. The first is that edges can be missing or broken, and the second is that extraneous edges can be found. The vertex proximity module can make only two types of mistakes. Junctions that should have been grouped can be separated, and junctions that should have been separated can be grouped. The continuity module can make only two errors as well. Either an extra break can be added, or a corner can be missed. The junction typing algorithm also has two types of errors. An error can be caused by a mistake in tangents. Mistaking a three tangent junction for an arrow junction is an example of this error. Mistakes in curvature can also cause errors. An example of this error is mistaking a Curvature-L junction for an phantom junction. 
In this set of images there are a total of 245 visible junctions and 323 visible arcs. There were a total of 113 errors found in the initial processing, so the majority of the visible junctions and arcs were properly processed. There was only one image with a perfect initial figure hypothesis, Image 8. There was also only one image with a very bad initial figure hypothesis, Image 7. The difficulties in Image 7 arise on the right hand side of the background block. The rightmost surface of this block is close to an accidental alignment and is therefore segmented into a large number of small surfaces that these algorithms have difficulty analyzing.

Appendix A shows the parameters that were used to calculate the initial figure hypotheses. Table 5 shows that the choice of parameters has balanced the errors. As an example, the value of the vertex proximity threshold results in approximately the same number of junctions incorrectly grouped (7) as broken (12). The same can be said for the continuity algorithm and the missing (13) and extraneous breaks (13). The weak membrane edge detection is also creating roughly the same number of extraneous edges (14) as missing edges (20).

The missing phantom junctions are not errors in the initial figure hypothesis. In Malik's line labeling paradigm [30], each curved arc was given a phantom junction. These phantom junctions were not included in the initial figure hypothesis because they can be detected from line labeling failures and placed only at junctions where difficulties arise.

One particular problem noticed in this processing was that all three tangent junctions were mistaken for arrow junctions. Almost all of the curvature-L junctions were mistaken for $\mathrm{L}$ junctions. This happens because finding the curvature near junctions is difficult. This type of problem can be ameliorated by the introduction of other modules such as shape from shading. Separate research is currently under way to address this problem.

Examples of the results of the diagnostic line labeling module are at the end of Section 5. A summary of the actual problems that exist in the test images is given in Table 6 . Table 7 shows a comparison between the faults that exist in the images and the ones that were diagnosed by the algorithm. Multiple diagnoses for a single image were attained by manually correcting problems as they were discovered to permit further problems to be diagnosed. Table 7 summarizes how well the problems found in the initial figure hypothesis have been detected by the modified line labeling paradigm using the floating object heuristic. Grouped junctions are easily detected since having more than three arcs at a junction is not permitted within the object domain under study in this paper.

Curvature and tangent mistakes in the classification of the junctions are also found well. The exception to this occurs when a pair of mistakes hide a problem. As an example, if a three tangent junction is mistakenly identified as an arrow and an adjacent curvature- $\mathrm{L}$ junction is also 
mistaken for an L junction, then the mistake cannot be diagnosed since the labeling produced is in fact legal in this object domain. In this case, the limb edge will be found to be a jump edge. While this is unfortunate, it should be noted that both limb edges and jump edges represent discontinuities of the scene depth and it is this similarity that permits confusion.

The mistakes that are the most difficult to diagnose are the missing breaks and extraneous breaks in continuity. The difficulty with diagnosis is that usually labelings both with or without breaks are legal. Although the problem in these cases cannot be diagnosed, it should be noted that the line labeling that is obtained is correct. In fact, the correctness of the line labeling without regard to the placement of these breaks is what prevents proper diagnosis.

The localization of the diagnosis of problems in the figure hypothesis is important. In almost all of the cases reported in Table 6 the problem was diagnosed at the correct junction. In three cases, the problem was diagnosed at an adjacent junction.

The modified line labeling algorithm has been shown to be useful for identifying problems in the initial line labeling hypothesis, although there are problems which cannot be diagnosed. In all cases in the test bed of images, the junction that was diagnosed as being the problem was either was the problem vertex, or was adjacent to the problem vertex.

Finally, we give the results of the corrections of the problems which were identified by the diagnostics of the line labeling problem. Table 8 gives a summary of the results from this module.

A comparison can be made between the problems that have been diagnosed in Section 5 and the problems that can be repaired using the repair knowledge sources. This comparison is shown in Table 9. This table shows that most of the diagnosed problems can be fixed using the repair knowledge sources. The extraneous edge problem that was not fixed was in image 7 where a near accidental alignment lead to an excessively cluttered area of the image. The overall performance of this system in detecting correct line labelings will be discussed in Section 8 .

Of the twenty-two images in the test bed given in Appendix B, one image could not be labeled, one image had a convex edge mistaken for a limb, and five images had limb edges mistaken for jump edges, and one image had a missing arc that wasn't replaced. Therefore fourteen of the twenty-two images were processed completely correctly. Of the eight failures, only one produced no line labeling. In the other seven failures, only one or two arcs were mislabeled. Integration and diagnosis have dramatically improved the initial figure hypotheses, as can be seen in Table 10 . 


\subsection{Execution Timings of Algorithms}

The algorithms discussed in this paper were implemented in LISP, C, and Fortran on a Sparcserver 690MP. The majority of the implementation was done in Allegro Common Lisp version 3.1.4, using the GBB blackboard system shell version 1.2. The LISP was compiled before timings were done. $\mathrm{C}$ was interfaced to Lisp to improve the execution speed of the numerical parts of the program. Most array operations were implemented in $\mathrm{C}$, as was the energy minimization in the continuity algorithm. Fortran was only used for the program that performs the Voronoi tesselation.

The run time of the edge detection, thinning and connected components algorithms are all independent of the contents of the image, run times are given for one image (image 2 in the test set in Appendix B). The weak membrane edge detection ran in 214 seconds (3.5 minutes). The thinning took .7 seconds. The connected components algorithm took 10.4 seconds. The longest time it took to process an image and generate a labeled line drawing after weak membrane edge detection, thinning, and connected components had been performed was 14.6 minutes on image 20. The run times of these algorithms were found using the time command in Lisp. All times exclude garbage collection.

\section{Conclusions}

This paper described the POLL system for obtaining labeled line drawings from single intensity images using an integrated system. The goal of this research was to demonstrate that even when individual visual processing modules are not very robust, one can increase the robustness and quality of the overall system and $3 D$ interpretation by integrating a collection of such modules.

In particular, the POLL system has demonstrated the following capabilities:

1. One can obtain $3 D$ interpretations of real images (single intensity images) in the form of labeled line drawings.

2. This system is unique in that it uses piecewise smooth objects, instead of the more restrictive planar or quadric surface models that are often used, particularly in range imagery as in $[62,35]$. This enhances the generality of the system, but also greatly increases the difficulty of the problem.

3. This result is achieved through the integration of the following modules: edge detection, region segmentation, curvilinear grouping, and line labeling.

4. In the cases where an unambiguous labeling cannot be extracted, the POLL system will 
produce partial results. These partial results can be further refined and disambiguated as other modules are added and further interpretations can be done. Examples of such modules can be shape from shading, stereo, etc.

5. The POLL system has demonstrated that the blackboard systems are useful platforms for integrating different visual modules with diverse representations.

6. The system also works without reference to a set of object models. This distinguishes the system from efforts like the Schema blackboard system [37], where object models were extensively used.

One of the shortcomings of the POLL system is that it does require objects to have smooth surfaces with no markings and texture. This is a direct result of the curvilinear line labeling scheme used to interpret the line drawings. Future research will look into the integration of modules that can process such objects. The shape from shading and symmetry detection can be incorporated in the future which would improve the results. The problem of adding shape from shading and stereo modules to this scheme is being studied independently and the initial

results in terms of the ability to distinguish limb edges from jump edges are encouranging [63]. Other modules that could be added are surface fitting modules which would guide the $3 D$ interpretation process more robustly.

\section{A Parameters}

Many of the modules and algorithms used in this paper have parameters. These parameters were set empirically, after experimentation with the test bed of images which will be shown in Appendix B. In order to make this work reproducable, all of the parameters used are given in this appendix. The parameters are in the order of first discussion.

Section 3.2 states that the continuity grouping algorithm of Trytten and Tuceryan [21] is used to perform the curvilinear grouping. The parameters for this algorithm fall into three categories: parameters for the energy formulation, parameters for Canny edge detection, and the multi-grid relaxation parameters. These parameters and their physical interpretations are summarized in Table 11. 
Table 4: The knowledge sources used to create the line drawing and possible errors made.

\begin{tabular}{|c|c|}
\hline Module & Error \\
\hline \hline Edge Detection & False Edges \\
\hline & Missing Edges \\
\hline \hline Continuity & False Corners \\
\hline \hline Vertex Proximity & Missing Corners \\
\hline & Not enough grouping \\
\hline \hline Junction Typing & Wrong type \\
\hline & Wrong class \\
\hline & Phantom vertex missing \\
\hline
\end{tabular}


Table 5: The results of processing the test bed of images shown in Appendix B are shown below. ME stands for missing edges. EE is extraneous edges. GJ are grouped junctions. BJ are broken junctions. MB are missed breaks. EB are extraneous breaks. CM are curvature mistakes. TM are tangent mistakes. MP are missing phantom junctions. These nine failures summarize all of the problems found in the test bed of images. Image 7 had many extraneous edges that were difficult to count, as signified by the a.

\begin{tabular}{|c|c|c|c|c|c|c|c|c|c|}
\hline \multicolumn{10}{|c|}{ Initial Line Labeling Problems } \\
\hline Image & $\mathrm{ME}$ & $\mathrm{EE}$ & GJ & BJ & MB & EB & $\mathrm{CM}$ & $\mathrm{TM}$ & MP \\
\hline 1 & 0 & 0 & 0 & 0 & 0 & 2 & 0 & 1 & 1 \\
\hline 2 & 1 & 0 & 0 & 0 & 0 & 0 & 0 & 0 & 0 \\
\hline 3 & 0 & 0 & 1 & 0 & 0 & 0 & 1 & 2 & 0 \\
\hline 4 & 4 & 0 & 2 & 0 & 1 & 1 & 0 & 1 & 0 \\
\hline 5 & 0 & 0 & 0 & 2 & 0 & 0 & 0 & 0 & 0 \\
\hline 6 & 0 & 1 & 0 & 0 & 0 & 0 & 0 & 2 & 0 \\
\hline 7 & 2 & $\mathrm{a}$ & 0 & 1 & 0 & 3 & 0 & 0 & 0 \\
\hline 8 & 0 & 0 & 0 & 0 & 0 & 0 & 0 & 0 & 0 \\
\hline 9 & 0 & 0 & 0 & 0 & 0 & 1 & 0 & 1 & 0 \\
\hline 10 & 0 & 0 & 0 & 1 & 2 & 1 & 0 & 2 & 0 \\
\hline 11 & 1 & 0 & 0 & 0 & 0 & 1 & 0 & 0 & 0 \\
\hline 12 & 1 & 0 & 0 & 0 & 1 & 0 & 0 & 0 & 0 \\
\hline 13 & 0 & 1 & 0 & 0 & 1 & 3 & 1 & 0 & 0 \\
\hline 14 & 0 & 0 & 0 & 1 & 0 & 2 & 0 & 2 & 0 \\
\hline 15 & 3 & 0 & 0 & 1 & 2 & 0 & 0 & 1 & 0 \\
\hline 16 & 3 & 0 & 0 & 0 & 0 & 1 & 1 & 1 & 0 \\
\hline 17 & 2 & 0 & 1 & 1 & 2 & 0 & 0 & 2 & 0 \\
\hline 18 & 3 & 0 & 0 & 1 & 1 & 1 & 0 & 0 & 1 \\
\hline 19 & 0 & 2 & 0 & 1 & 1 & 3 & 1 & 0 & 0 \\
\hline 20 & 0 & 2 & 3 & 0 & 0 & 3 & 2 & 1 & 0 \\
\hline 21 & 0 & 1 & 0 & 3 & 2 & 0 & 0 & 0 & 0 \\
\hline 22 & 0 & 7 & 0 & 1 & 0 & 4 & 1 & 0 & 0 \\
\hline Total & 20 & 14 & 7 & 13 & 13 & 26 & 7 & 16 & 2 \\
\hline
\end{tabular}


Table 6: A listing of the problems in the test bed of images from Appendix B showing which problems are diagnosed. ME stands for missing edges. EE is extraneous edges. GJ are grouped junctions. BJ are broken junctions. MB are missed breaks. EB are extraneous breaks. CM are curvature mistakes. TM are tangent mistakes. MP are missing phantom junctions. Notation a means that the problem diagnosis was found at a vertex adjacent to the vertex where the problem actually existed. In Image 7, the notation b was used to indicate that one of the problems was correctly diagnosed, but the image was too cluttered to be fixed using the methods of this paper.

\begin{tabular}{|l||c|c||c|c||c|c||c|c||c|}
\hline \multicolumn{1}{|c||}{} & \multicolumn{7}{c|}{ Diagnosis } \\
\hline Image & ME & EE & GJ & BJ & MB & EB & CM & TM & MP \\
\hline 1 & 0 & 0 & 0 & 0 & 0 & 0 & 0 & 0 & 1 \\
\hline 2 & 1 & 0 & 0 & 0 & 0 & 0 & 0 & 0 & 0 \\
\hline 3 & 0 & 0 & 1 & 0 & 0 & 0 & 1 & 2 & 0 \\
\hline 4 & 3 & 0 & 2 & 0 & 0 & 0 & 0 & 1 & 0 \\
\hline 5 & 0 & 0 & 0 & $1 \mathrm{a}$ & 0 & 0 & 0 & 0 & 0 \\
\hline 6 & 0 & 1 & 0 & 0 & 0 & 0 & 0 & 2 & 0 \\
\hline $7 \mathrm{~b}$ & & & & & & & & & \\
\hline 8 & 0 & 0 & 0 & 0 & 0 & 0 & 0 & 0 & 0 \\
\hline 9 & 0 & 0 & 0 & 0 & 0 & 0 & $\mathrm{~b}$ & 0 & 1 \\
\hline 10 & 0 & 0 & 0 & 0 & 0 & 0 & 0 & $1 \mathrm{a}$ & 0 \\
\hline 11 & 1 & 0 & 0 & 0 & 0 & 0 & 0 & 0 & 0 \\
\hline 12 & 1 & 0 & 0 & 0 & 0 & 0 & 0 & 0 & 0 \\
\hline 13 & 0 & 1 & 0 & 0 & 0 & 0 & 1 & 0 & 0 \\
\hline 14 & 0 & 0 & 0 & 1 & 0 & 1 & 0 & 0 & 0 \\
\hline 15 & 1 & 0 & 0 & 1 & 0 & 0 & 0 & 1 & 0 \\
\hline 16 & 3 & 0 & 0 & 0 & 0 & $1 \mathrm{a}$ & 1 & 1 & 0 \\
\hline 17 & 0 & 0 & 1 & 1 & 0 & 0 & 0 & 2 & 0 \\
\hline 18 & 2 & 0 & 0 & 1 & 0 & 0 & 0 & 0 & $1 \mathrm{a}$ \\
\hline 19 & 0 & 3 & 0 & 0 & 0 & 0 & $1 \mathrm{a}$ & 0 & 0 \\
\hline 20 & 0 & 2 & 3 & 0 & 0 & 0 & 2 & 0 & 0 \\
\hline 21 & 0 & 1 & 0 & 2 & 0 & 0 & 0 & 0 & 0 \\
\hline 22 & 0 & 3 & 0 & 1 & 0 & 1 & 1 & 1 & 0 \\
\hline Total & 12 & 11 & 7 & 8 & 0 & 3 & 7 & 12 & 2 \\
\hline
\end{tabular}


Table 7: A comparison between the faults in the images from Table 5, and the problems that were diagnosed using the floating object heuristic.

\begin{tabular}{|l|c|c|c|c|c|c|c|c|c|}
\hline Source & ME & EE & GJ & BJ & MB & EB & CM & TM & MP \\
\hline Initial & 20 & 14 & 7 & 13 & 13 & 26 & 7 & 16 & 2 \\
\hline Diagnosed & 12 & 11 & 7 & 8 & 0 & 3 & 7 & 11 & 2 \\
\hline
\end{tabular}


Table 8: A listing of the problems in the test bed of images from Appendix B showing which problems are repaired. ME stands for missing edges. EE is extraneous edges. GJ are grouped junctions. BJ are broken junctions. MB are missed breaks. EB are extraneous breaks. CM are curvature mistakes. TM are tangent mistakes. MP are missing phantom junctions. Notation a indicates a correction that was manually made that may be beyond the scope of the repair knowledge sources discussed in this section.

\begin{tabular}{|l||c|c||c|c||c|c||c|c||c|}
\hline \multicolumn{1}{|c||}{} & \multicolumn{7}{c|}{ Repair } \\
\hline Image & ME & EE & GJ & BJ & MB & EB & CM & TM & MP \\
\hline 1 & 0 & 0 & 0 & 0 & 0 & 0 & 0 & 0 & 1 \\
\hline 2 & 1 & 0 & 0 & 0 & 0 & 0 & 0 & 0 & 0 \\
\hline 3 & 0 & 0 & 1 & 0 & 0 & 0 & 1 & 2 & 0 \\
\hline 4 & 3 & 0 & 2 & 0 & 0 & 0 & 0 & 1 & 0 \\
\hline 5 & 0 & 0 & 0 & 1 & 0 & 0 & 0 & 0 & 0 \\
\hline 6 & 0 & 1 & 0 & 0 & 0 & 0 & 0 & 2 & 0 \\
\hline $7 \mathrm{a}$ & & & & & & & & & \\
\hline 8 & 0 & 0 & 0 & 0 & 0 & 0 & 0 & 0 & 0 \\
\hline 9 & 0 & 0 & 0 & 0 & 0 & 0 & 0 & 1 & 0 \\
\hline 10 & 0 & 0 & 0 & 0 & 0 & 0 & 0 & 0 & 0 \\
\hline 11 & 1 & 0 & 0 & 0 & 0 & 0 & 0 & 0 & 0 \\
\hline 12 & 1 & 0 & 0 & 0 & 0 & 0 & 0 & 0 & 0 \\
\hline 13 & 0 & 1 & 0 & 0 & 0 & 0 & $1 \mathrm{a}$ & 0 & 0 \\
\hline 14 & 0 & 0 & 0 & 1 & 0 & 1 & 0 & 0 & 0 \\
\hline 15 & 1 & 0 & 0 & 1 & 0 & 0 & 0 & 1 & 0 \\
\hline 16 & 3 & 0 & 0 & 0 & 0 & 0 & 1 & 1 & 0 \\
\hline 17 & 0 & 0 & 1 & 1 & 0 & 0 & 0 & 2 & 0 \\
\hline 18 & 2 & 0 & 0 & 1 & 0 & 0 & 0 & 0 & 1 \\
\hline 19 & 0 & 3 & 0 & 0 & 0 & 0 & 0 & 0 & 0 \\
\hline 20 & 0 & 2 & 3 & 0 & 0 & 0 & 2 & 0 & 0 \\
\hline 21 & 0 & 1 & 0 & 2 & 0 & 0 & 0 & 0 & 0 \\
\hline 22 & 0 & 3 & 0 & 1 & 0 & 1 & 1 & 0 & 0 \\
\hline Total & 12 & 11 & 7 & 8 & 0 & 2 & 6 & 10 & 2 \\
\hline
\end{tabular}


Table 9: A comparison between diagnosed problems and repaired problems in line labelings.

\begin{tabular}{|l|c|c|c|c|c|c|c|c|c|}
\hline Source & ME & EE & GJ & BJ & MB & EB & CM & TM & MP \\
\hline Diagnosis & 12 & 11 & 7 & 8 & 0 & 3 & 7 & 12 & 2 \\
\hline Repair & 12 & 11 & 7 & 8 & 0 & 2 & 6 & 10 & 2 \\
\hline
\end{tabular}

Table 10: The result of the line labeling process. A "Yes" in the intial column means that a proper line labeling was found from the initial figure hypothesis. A "Yes" in the final column means that a correct line labeling was found after correction. An "A" in the final column means that the line labeling was correct except for a single error. A "B" means that a missing arc wasn't replaced. A "No" in a column means that no correct line labeling was found.

\begin{tabular}{|c|c|c|}
\hline Image & Initial & Final \\
\hline 1 & No & Yes \\
\hline 2 & No & Yes \\
\hline 3 & No & Yes \\
\hline 4 & No & Yes \\
\hline 5 & No & Yes \\
\hline 6 & No & Yes \\
\hline 7 & No & No \\
\hline 8 & Yes & Yes \\
\hline 9 & No & Yes \\
\hline 10 & No & A \\
\hline 11 & No & Yes \\
\hline 12 & No & Yes \\
\hline 13 & No & Yes \\
\hline 14 & No & A \\
\hline 15 & No & B \\
\hline 16 & No & Yes \\
\hline 17 & No & $A$ \\
\hline 18 & No & Yes \\
\hline 19 & No & Yes \\
\hline 20 & No & A \\
\hline 21 & No & $A$ \\
\hline 22 & No & A \\
\hline
\end{tabular}


Table 11: The parameters for the continuity grouping algorithm of [21].

\begin{tabular}{|c|c|c|}
\hline Algorithm & Parameter & Value \\
\hline \multirow[t]{4}{*}{ Canny edge detection } & $\sigma$ & 2 \\
\hline & mask size & 17 \\
\hline & low threshold & .60 \\
\hline & high threshold & .75 \\
\hline \multirow[t]{4}{*}{ Continuity } & $\beta$ & .28 \\
\hline & $\lambda$ & 1.0 \\
\hline & $\mu$ & 2.5 \\
\hline & length ratio & .30 \\
\hline \multirow[t]{5}{*}{ Multi-grid relaxation } & number of resolutions & 3 \\
\hline & number of $\mathrm{mg}$ iterations & 1 \\
\hline & $\epsilon$ & .001 \\
\hline & maximum iterations & 10 \\
\hline & step size & .20 \\
\hline
\end{tabular}


In Section 4.2.1, edge detection was performed using the weak membrane edge model and the GNC algorithm for energy minimization. The parameters for the edge detection are shown in Table 12. Curve hypotheses were created for the boundary of regions that were sufficiently large, the specific numerical definition of large is given in Table 12. Edgels that were too short were removed from consideration. The parameter for this is also given in Table 12.

Section 4.2.2 also used a variety of parameters. The circular arc fitting parameters were used to determine how many pixels near the endpoint of a curve should be used for approximating the curvature and tangent direction. The curvature values were found to be unreliable, and were assigned to three classes: straight, curved and very curved. These symbolic curvatures were used in all comparisons.

In Section 6.1, a parameter for the number of pixels that separate a line from an arc is discussed. The value for this parameter is three pixels.

\section{B Test Bed of Images}

The POLL system was tested on twenty-two intensity images captured using a Macintosh II-FX in the PRIP Laboratory of Michigan State University. These images are labeled and shown in Figure 15, Figure 16, Figure 17, and Figure 18. Most of the side surfaces of the bath tub toys used in Images 1 to 18 have shrunk and are slightly curved. 
Table 12: The parameters from Section 4.2.1

\begin{tabular}{|l|c|c|}
\hline Algorithm & Parameter & Value \\
\hline \multirow{3}{*}{ GNC } & $\lambda$ & 4 \\
\cline { 2 - 3 } & $h_{0}$ & 20 \\
\cline { 2 - 3 } & number of iterations & 20 \\
\hline Curve creation & Small regions & 20 pixels \\
\hline Curve creation & Small edgels & 5 pixels \\
\hline
\end{tabular}

Table 13: The parameter values used in Section 4.2.2.

\begin{tabular}{|l|c|c|}
\hline Algorithm & Parameter & Value \\
\hline Vertex proximity & closeness & 7 pixels \\
\hline \multirow{2}{*}{ Circular arc fitting } & minimum pixels & 5 pixels \\
\cline { 2 - 3 } & maximum pixels & 25 pixels \\
\hline \multirow{2}{*}{ Linear fitting } & minimum pixels & 2 pixels \\
\cline { 2 - 3 } & maximum pixels & 25 pixels \\
\hline \multirow{3}{*}{ Vertex typing } & tangent threshold & 20 degrees \\
\cline { 2 - 3 } & straight radius of curvature & more than 1000 \\
\cline { 2 - 3 } & curved radius of curvature & 100 to 1000 \\
\cline { 2 - 3 } & very curved radius of curvature & less than 100 \\
\hline
\end{tabular}




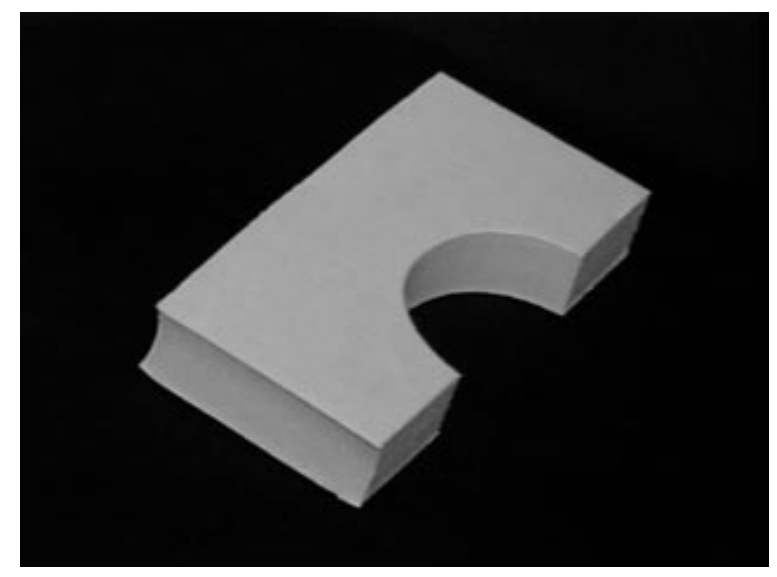

(a)

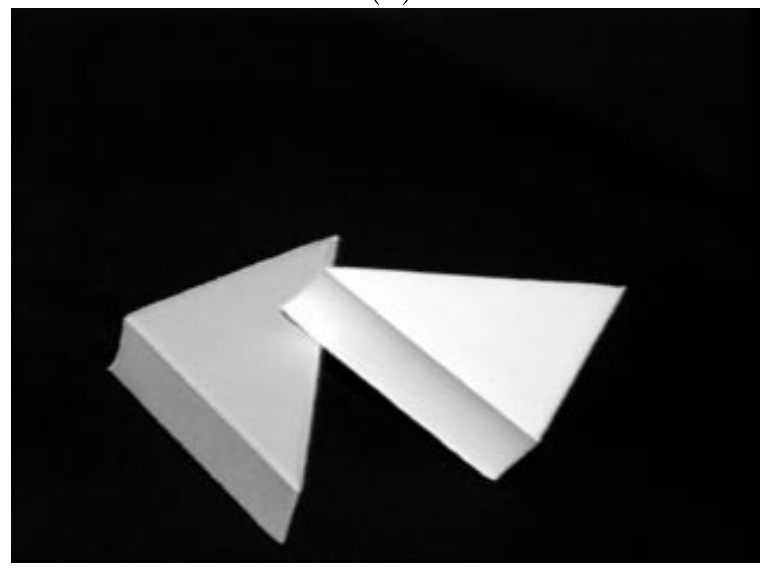

(c)

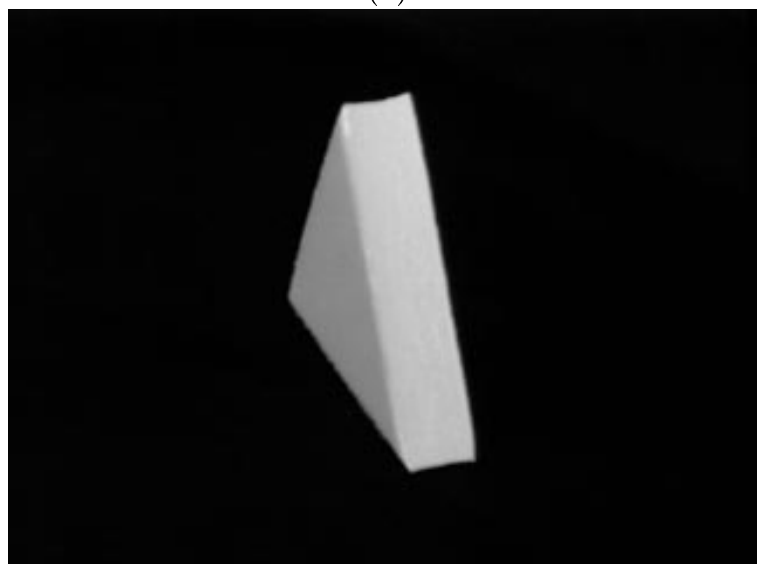

(e)

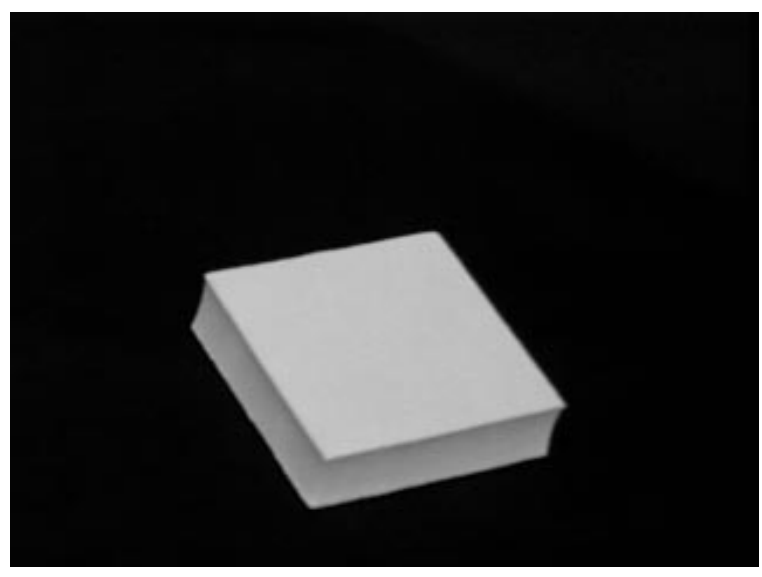

(b)

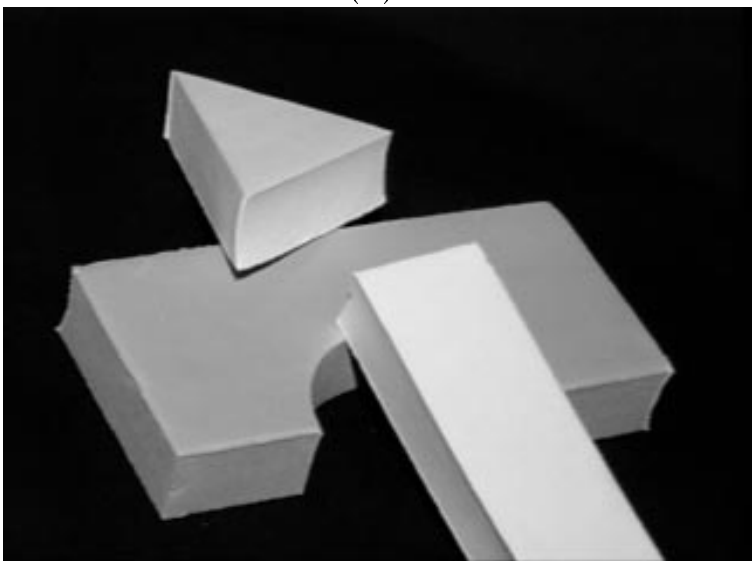

(d)

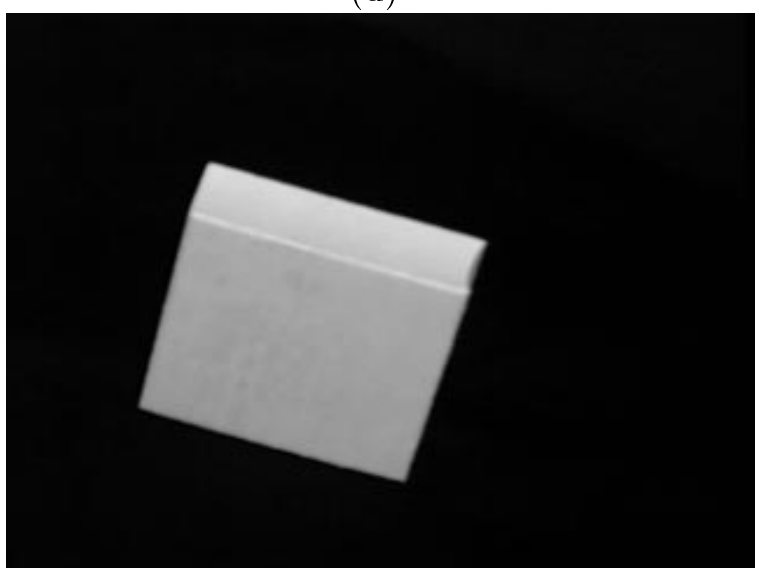

(f)

Figure 15: (a) Image 1. (b) Image 2. (c) Image 3. (d) Image 4. (e) Image 5. (f) Image 6. 


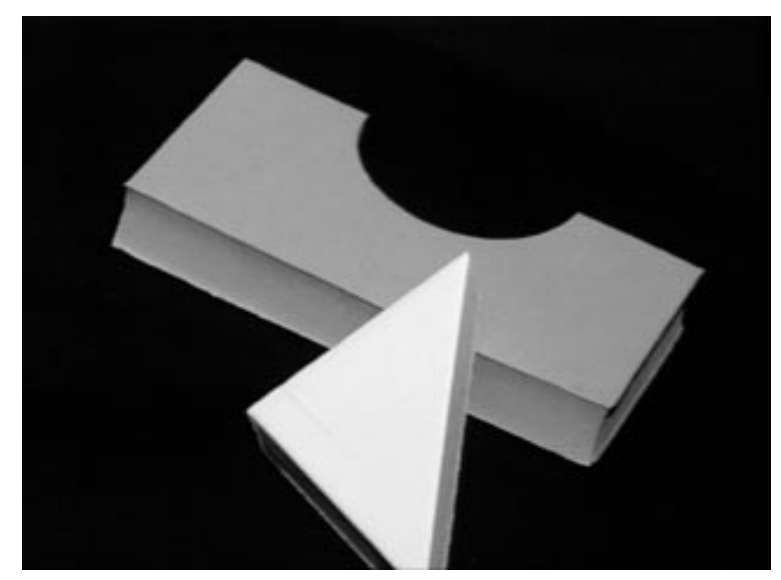

(a)

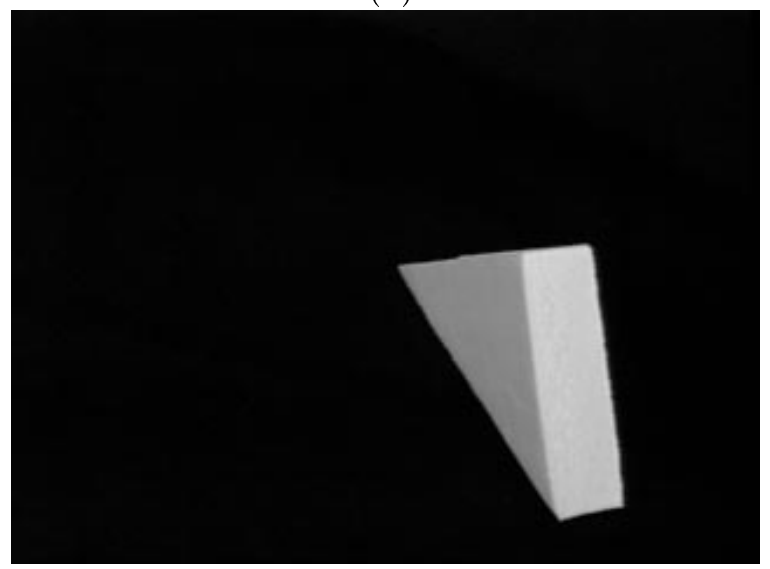

(c)

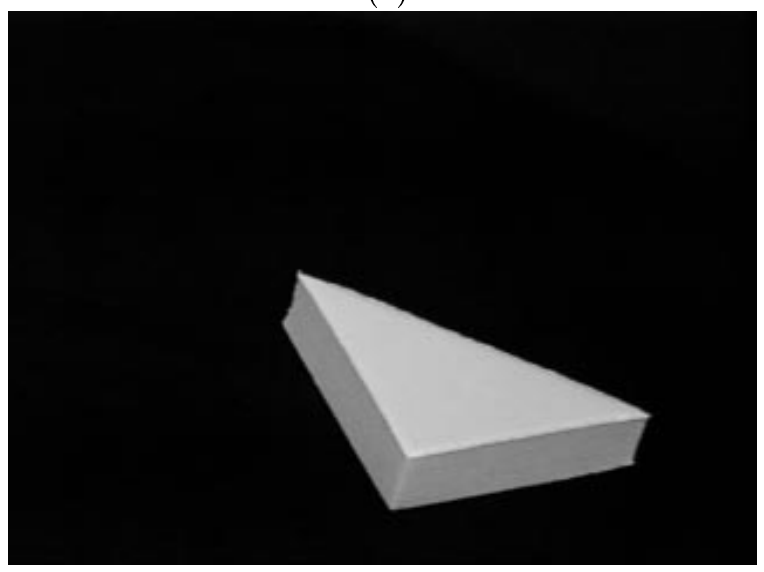

(e)

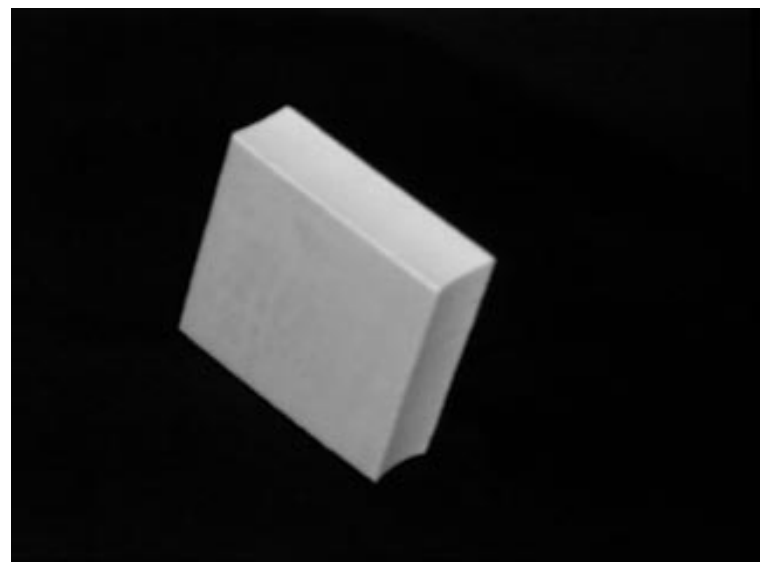

(b)

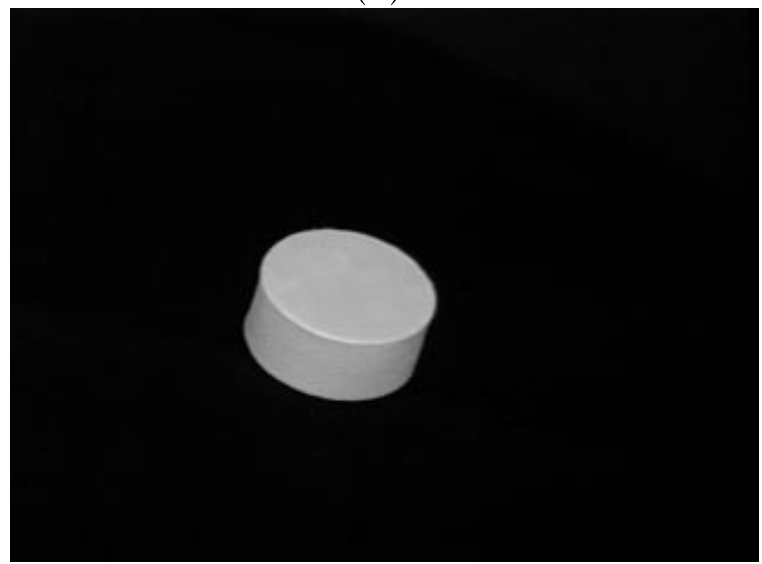

(d)

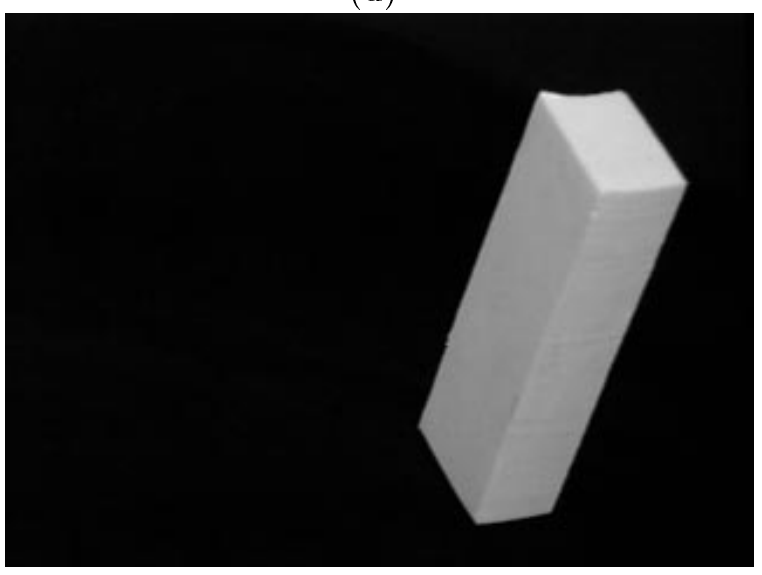

(f)

Figure 16: (a) Image 7. (b) Image 8. (c) Image 9. (d) Image 10. (e) Image 11. (f) Image 12. 


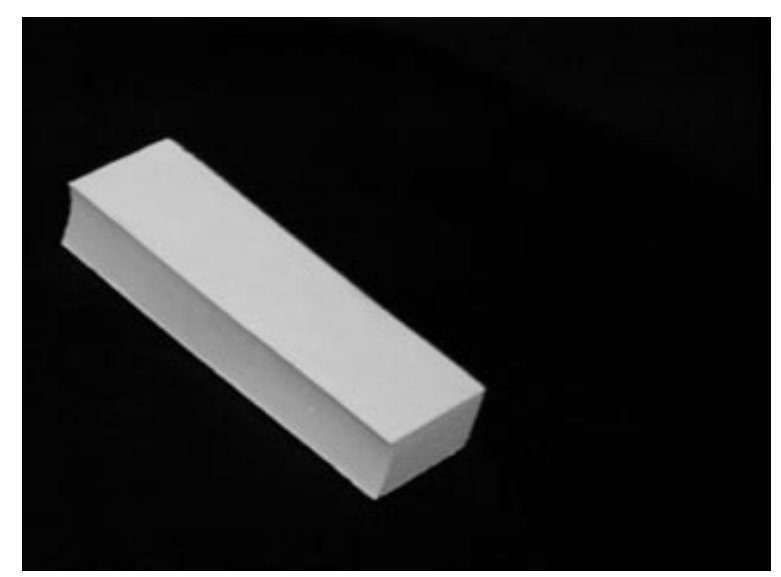

(a)

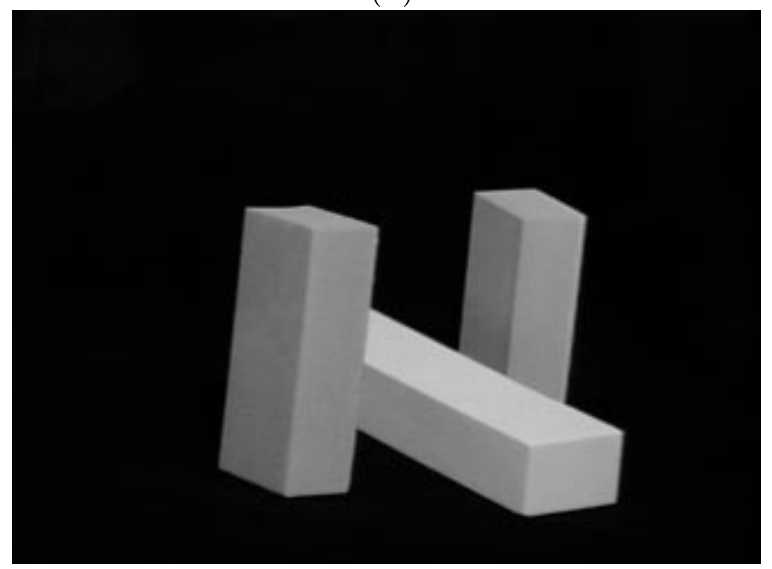

(c)

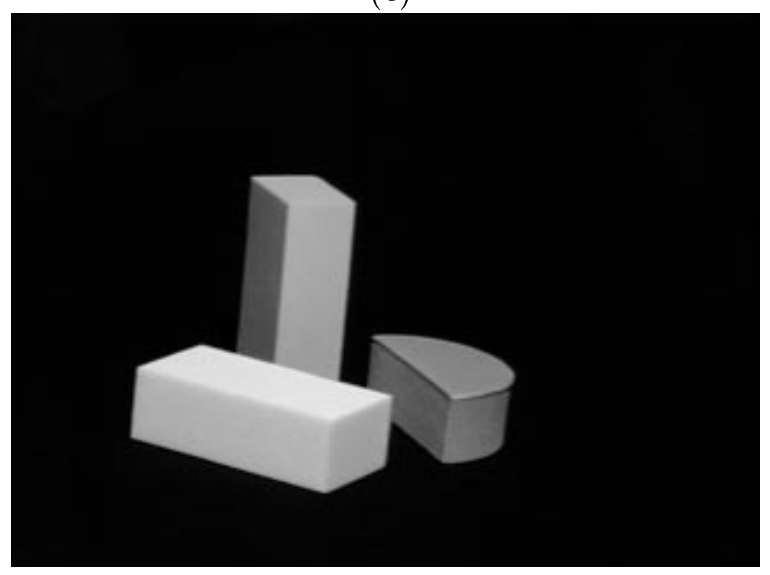

(e)

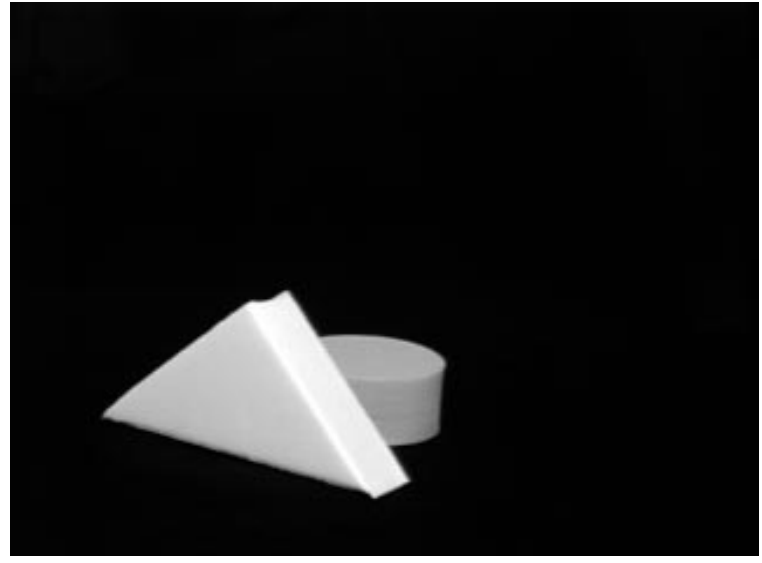

(b)

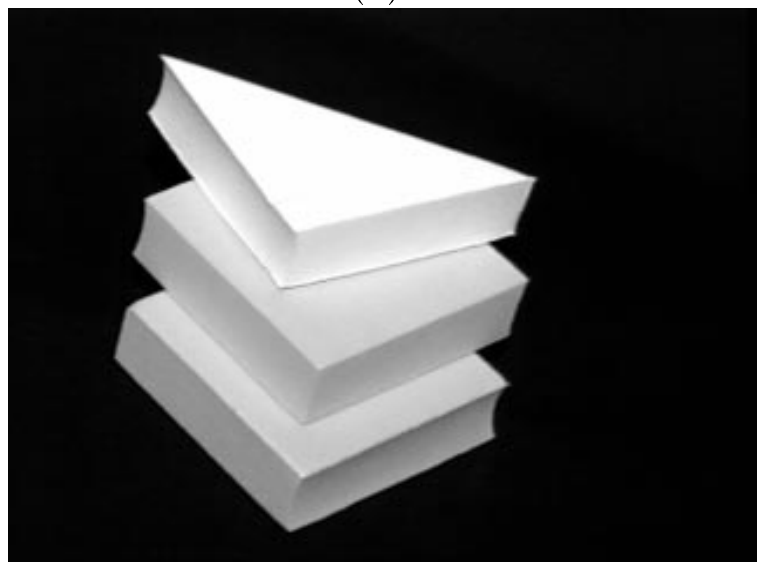

(d)

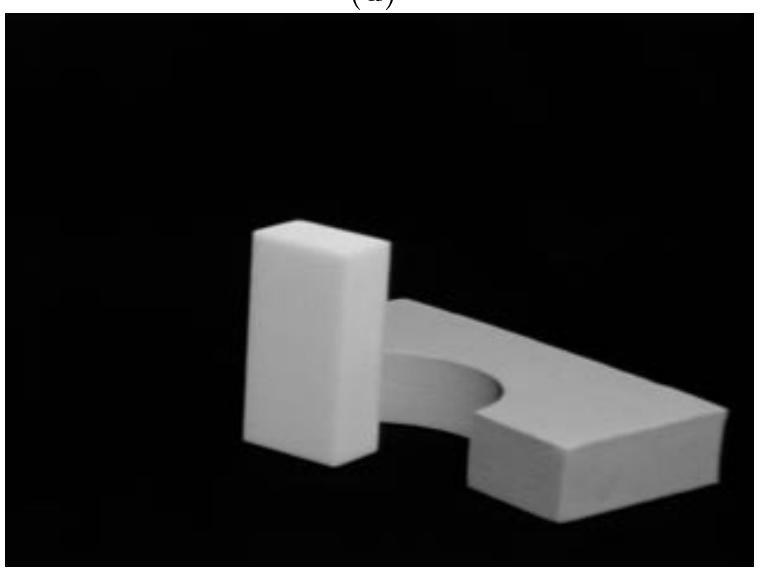

(f)

Figure 17: (a) Image 13. (b) Image 14. (c) Image 15. (d) Image 16. (e) Image 17. (f) Image 18. 


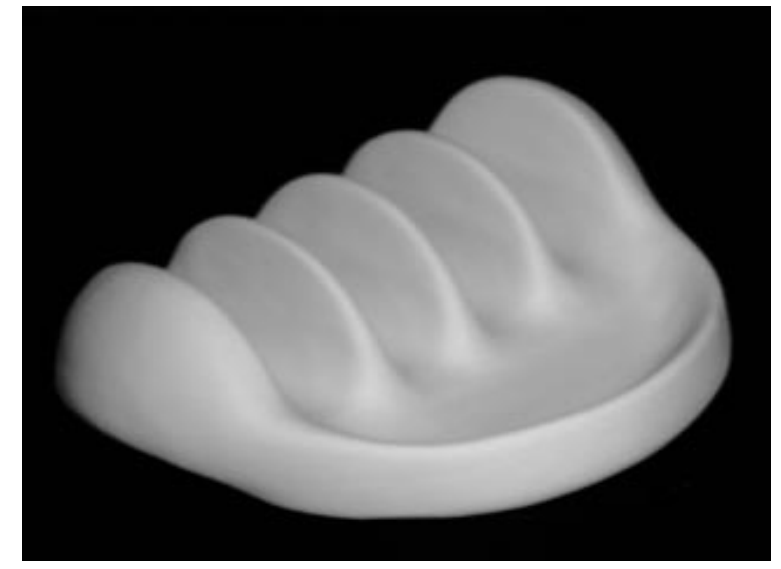

(a)

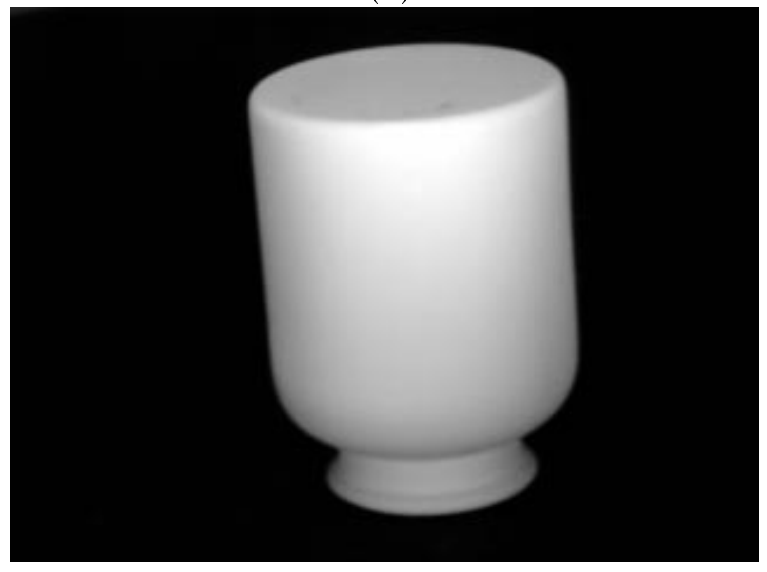

(c)

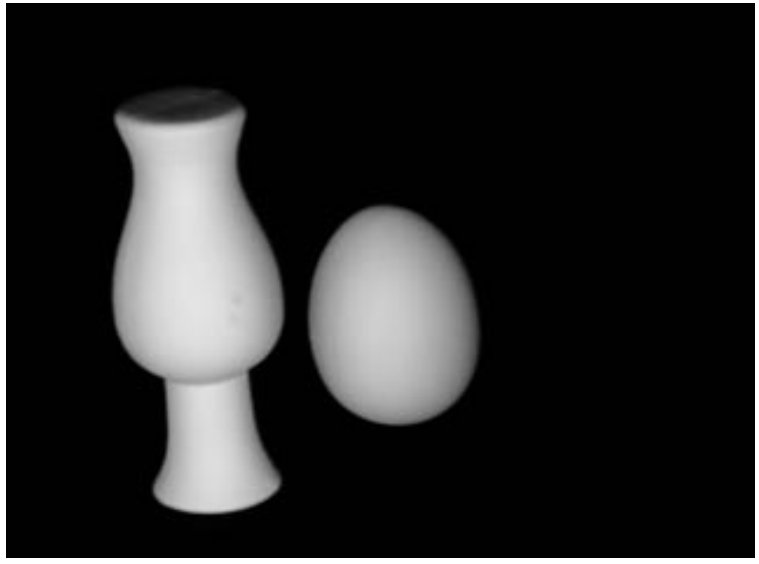

(b)

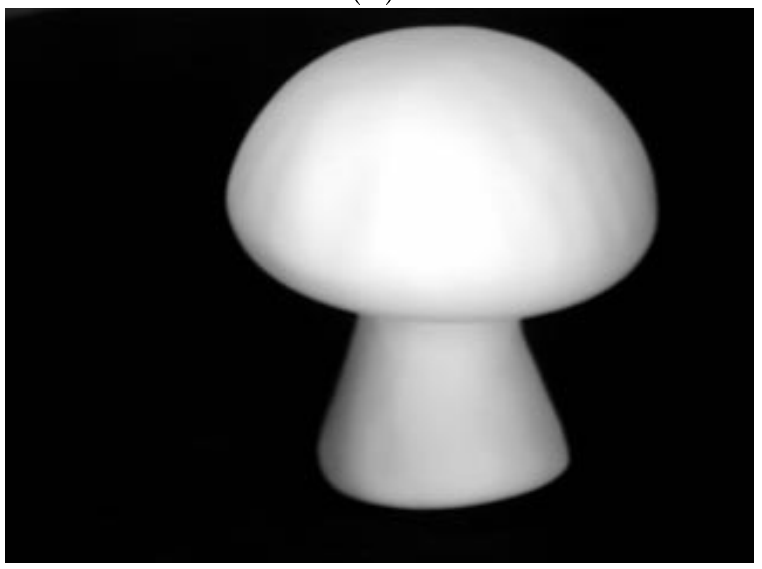

(d)

Figure 18: (a) Image 19. (b) Image 20. (c) Image 21. (d) Image 22. 


\section{References}

[1] F. P. Ferrie and M. D. Levine. Where and why local shading works. IEEE Transactions on Pattern Analysis and Machine Intelligence, 11(2):198-206, February 1989.

[2] R. T. Frankot and R. Chellappa. A method for enforcing integrability in shape from shading algorithms. In B. K. P. Horn and M. J. Brooks, editors, Shape from Shading, chapter 5, pages 89-122. The MIT Press, 1989.

[3] B. K. P. Horn. Obtaining shape from shading information. In Shape from Shading, pages 123-173. The MIT Press, Cambridge, Massachusetts, 1989.

[4] A. P. Pentland. Local shading analysis. In B. K. P. Horn and M. J. Brooks, editors, Shape from Shading, chapter 15, pages 443-488. Massachusetts Institute of Technology, Cambridge, Massachusetts, 1989.

[5] D. Blostein. Recovering the Orientation of Textured Surfaces in Natural Scenes. PhD thesis, University of Illinois, 1987.

[6] D. Blostein and N. Ahuja. Shape from texture: Integrating texture-element extraction and surface estimation. IEEE Transactions on Pattern Analysis and Machine Intelligence, 11(12):1233-1251, December 1989.

[7] L. G. Brown and H. Shvaytser. Surface orientation from projective foreshortening of isotropic texture autocorrelation. In Proceedings of IEEE Computer Vision and Pattern Recognition Society, pages 510-515, Ann Arbor, Michigan, 1988.

[8] Y. C. Jau and R. T. Chin. Shape from texture using the Wigner distribution. In Proceedings of the IEEE Computer Society Conference on Computer Vision and Pattern Recognition, pages 515-523, Ann Arbor, Michigan, June 1988.

[9] J. Aloimonos and D. Shulman. Integration of Visual Modules. Academic Press, San Diego, California, 1989.

[10] A. Blake, A. Zisserman, and G. Knowles. Surface descriptions from stereo and shading. In B. K. P. Horn and M. J. Brooks, editors, Shape from Shading, chapter 2, pages 29-52. Massachusetts Institute of Technology, Cambridge, Massachusetts, 1989.

[11] B. Kim and P. Burger. Calculation of surface position and orientation using the photometric stereo method. In Proceedings of IEEE Computer Vision and Pattern Recognition Society, pages 492-498, Ann Arbor, Michigan, 1988.

[12] I. Biederman. Human image understanding: Recent research and a theory. Computer Vision, Graphics, and Image Processing, 32:29-73, 1985.

[13] Irvin Rock. The Logic of Perception. The MIT Press, Cambridge, Massachusetts, 1982.

[14] David G. Lowe. Perceptual Organization and Visual Recognition. Kluwer Academic Publishers, Boston, 1985.

[15] D. H. Ballard and C. M. Brown. Computer Vision. Prentice Hall, New Jersey, 1982.

[16] M. D. Levine. Vision in Man and Machine. Computer Engineering Series. McGraw Hill, New York, 1985.

[17] Max Wertheimer. Laws of organization in perceptual forms. In W. D. Ellis, editor, A Source Book of Gestalt Psychology. Harcourt Brace, New York, 1939. 
[18] A. P. Witkin and J. M. Tenenbaum. On the role of structure in vision. In J. Beck, B. Hope, and A. Rosenfeld, editors, Human and Machine Vision, pages 481-543. Academic Press, New York, 1983.

[19] M. Tuceryan. Extraction of Perceptual Structure in Dot Patterns. PhD thesis, University of Illinois, Urbana-Champaign, Illinois, 1986.

[20] P. L. Rosin and G. A. W. West. Detection and verification of surfaces of revolution by perceptual grouping. Pattern Recognition Letters, 13:453-461, June 1992.

[21] D. A. Trytten and M. Tuceryan. Segmentation and grouping of object boundaries using energy minimization. In IEEE Conference on Computer Vision and Pattern Recognition, pages 730-731, Mauii, Hawaii, June 1991.

[22] J. D. McCafferty. Human and Machine Vision, Computing Perceptual Organization. Ellis Horwood Series in Digital and Signal Processing. Ellis Horwood, New York, 1990.

[23] S. Sarkar and K. L. Boyer. A highly efficient computational structure for perceptual organization. Technical Report SAMPL-90-06, The Ohio State University, November 1990.

[24] R. Mohan and R. Nevatia. Perceptual organization for scene segmentation and description. IEEE Transactions on Pattern Analysis and Machine Intelligence, 14(6):616-635, June 1992.

[25] D. A. Huffman. Impossible objects as nonsense sentences. In B. Meltzer and D. M. Michie, editors, Machine Intelligence, volume 6, pages 295-323. Edinburgh University Press, 1971.

[26] M. B. Clowes. On seeing things. Artificial Intelligence, 2:79-116, 1971.

[27] A. K. Macworth. Interpreting pictures of polyhedral scenes. Artificial Intelligence, 4:121137, 1973.

[28] D. Waltz. Understanding line drawings of scenes with shadows. In P. H. Winston, editor, Psychology of Computer Vision, pages 19-91. McGraw-Hill, New York, NY, 1975.

[29] T. Kanade. A theory of origami world. Artificial Intelligence, 13:279-311, 1980.

[30] J. Malik. Interpreting line drawings of curved objects. International Journal of Computer Vision, 1:73-103, 1987.

[31] G. Falk. Interpretation of imperfect line data as a three dimensional scene. Artificial Intelligence, 3:101-144, 1972.

[32] K. Sugihara. Machine Interpretation of Line Drawings. The MIT Press Series in Artificial Intelligence. The MIT Press, Cambridge, Massachusetts, 1986.

[33] I. Chakravarty. A generalized line and junction labeling scheme with applications to scene analysis. IEEE Transactions on Pattern Analysis and Machine Intelligence, PAMI1(2):202-205, April 1979.

[34] I. Chakravarty. The use of Characteristic Views as a Basis for Recognition of ThreeDimensional Objects. PhD thesis, Rensselaer Polytechnic Institute, Troy, New York, 1982.

[35] Greg C. Lee. Reconstruction of Line Drawing Graphs for Objects with Quadric Surfaces using Wing Features. PhD thesis, Michigan State University, East Lansing, Michigan, 1992. 
[36] J. Aloimonos. Unification and integration of visual modules: An extension of the Marr paradigm. In Proceedings of the DARPA Image Understanding Workshop, pages 507-551, 1989.

[37] B. A. Draper, R. T. Collins, J. Brolio, A. R. Hanson, and E. M. Riseman. Issues in the development of a blackboard-based schema system for image understanding. In R. Engelmore and T. Morgan, editors, Blackboard Systems, chapter 8, pages 189-218. Addison Wesley, 1988 .

[38] E. B. Gamble, D. Geiger, T. Poggio, and D. Weinshall. Integration of vision modules and labeling of surface discontinuities. IEEE Transactions on Systems, Man, and Cybernetics, 19(6):1576-1581, November/December 1989.

[39] H. Moravec. Sensor fusion in certainty grids for mobile robots. AI Magazine, pages 61-74, Summer 1988.

[40] Sateesha G. Nadabar. Markov Random Field Contextual Models in Computer Vision. PhD thesis, Michigan State University, East Lansing, Michigan, 1992.

[41] S. Geman and D. Geman. Stochastic relaxation, Gibbs distributions, and Bayesian restoration of images. IEEE Transactions on Pattern Analysis and Machine Intelligence, PAMI6(6):721-741, November 1984.

[42] T. Poggio, V. Torre, and C Koch. Computational vision and regularization theory. Nature, 317:638-643, 1985.

[43] D. Terzopoulos. Regularization of inverse visual problems involving discontinuities. IEEE Transactions on Pattern Analysis and Machine Intelligence, 8(4):413-424, July 1986.

[44] A. Blake. Comparison of efficiency of deterministic and stochastic algorithms for visual reconstruction. IEEE Transactions on Pattern Analysis and Machine Intelligence, 11(1):212, January 1989.

[45] A. L. Abbott and N. Ahuja. Surface reconstruction by dynamic integration of focus, camera vergence, and stereo. In Proceedings of the Second International Conference on Computer Vision, pages 532-543, 1988.

[46] J. Malik and D. Maydan. Recovering three-dimensional shape from a single image of curved objects. IEEE Transactions on Pattern Analysis and Machine Intelligence, 11(6):555-566, June 1989.

[47] R. Engelmore and T. Morgan. Blackboard Systems. Addison Wesley, New York, 1988.

[48] L. D. Erman, F. Hayes-Roth, V. R. Lesser, and D. R. Reddy. The hearsay-II speechunderstanding system: Integrating knowledge to resolve uncertainty. In R. Engelmore and T. Morgan, editors, Blackboard Systems, chapter 3, pages 31-86. Addison Wesley, 1988.

[49] M. Nagao, T. Matsuyama, and H. Mori. Structural analysis of complex aerial photographs. In R. Engelmore and T. Morgan, editors, Blackboard Systems, chapter 9, pages 189-218. Addison Wesley, 1988.

[50] V. Jagannathan, R. Dodhiawala, and L. S. Baum. Blackboard Architectures and Applications. Harcourt Brace Jovanovich, Boston, 1989.

[51] A. Blake and A. Zisserman. Visual Reconstruction. The MIT Press, Cambridge, Massachusetts, 1987. 
[52] J. Canny. A computational approach to edge detection. IEEE Transactions on Pattern Analysis and Machine Intelligence, PAMI-8(6), November 1986.

[53] J. B. Burns, A. R. Hanson, and E. M. Riseman. Extracting straight lines. In M. A. Fischler and O. Firschein, editors, Readings in Computer Vision, Issues, Problems, Principles, and Paradigms, pages 180-183. Morgan Kaufmann, 1987.

[54] P. V. C. Hough. Method and means for recognizing complex patterns. U. S. Patent, no. 3069654, 1962.

[55] D. H. Ballard. Generalizing the Hough transform to detect arbitrary shapes. Pattern Recognition, 13:111-122, 1981.

[56] J. Illingworth and J. Kittler. A survey of the Hough transform. Computer Vision, Graphics, and Image Processing, 44:87-116, 1988.

[57] Narendra Ahuja. Dot pattern processing using Voronoi neighborhoods. IEEE Transactions on Pattern Analysis and Machine Intelligence, PAMI-4(3):336-343, May 1982.

[58] K. Q. Gallagher, D. D. Corkill, and P. M. Johnson. Gbb reference manual. Technical Report 88-66, University of Massachusetts at Amherst, Amherst, Massachusetts, July 1988.

[59] A. Rosenfeld and A. C. Kak. Digital Picture Processing. Academic Press, New York, 1976.

[60] W. H. Press, B. P. Flannery, S. A. Teukolsky, and W. T. Vetterling. Numerical Recipes in C: The Art of Scientific Computing. Cambridge University Press, 1988.

[61] P. H. Winston. Artificial Intelligence. Addison-Wesley, Reading, Mass., 2nd edition edition, 1984.

[62] Patrick J. Flynn. CAD-Based Computer Vision: Modeling and Recognition Strategies. PhD thesis, Department of Computer Science, Michigan State University, 1990.

[63] S. Pankanti, A. K. Jain, and M. Tuceryan. On integration of vision modules. In Proceedings of the Conference on Computer Vision and Pattern Recognition, Seattle, WA, 1994. IEEE. 